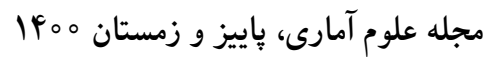

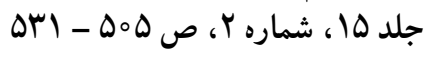

DOI: $10.29252 /$ jss.15.2.505

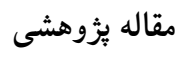

\section{مدلبندى باسخهاى طولى آميخته دومتغيره بيوسته-شمارشى و بيوسته-ترتيبى آماسيده با

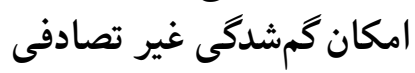

\author{
نسترن شريفيان، احسان بهرامى سامانى \\ كروه آمار، دانشكده علوم رياضى، دانشعاه شهيد بهشتى، تهران

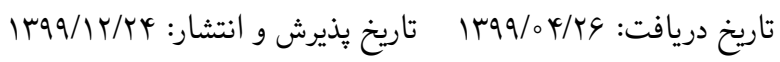

جكيده: يكى از مسائل مهم مطالعات طولى، دادههايى هستند كه به دليل گمشدن ملاقاتها يا انصراف

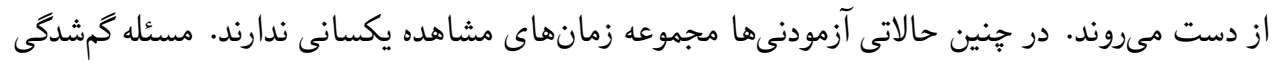

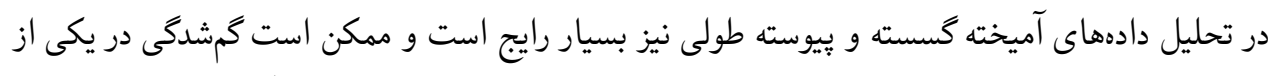

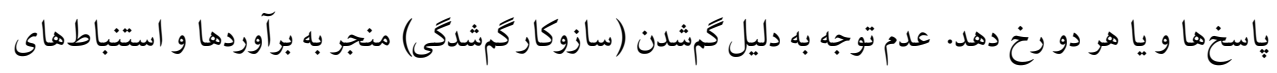

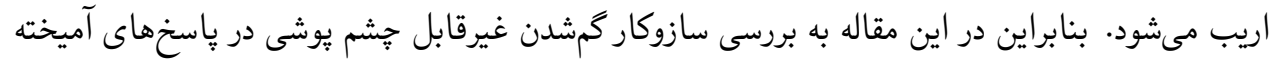

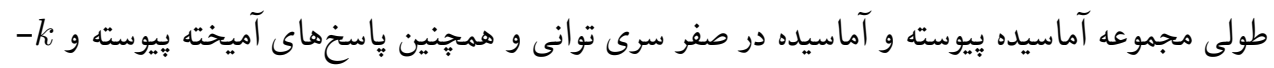

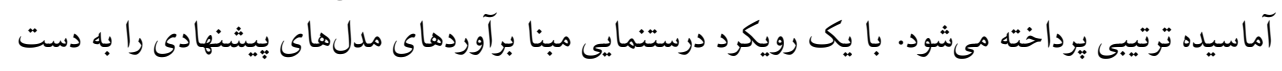

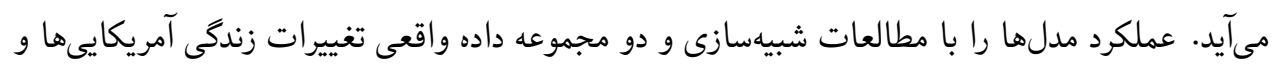
آزمون موفقيت فردى بيبادى مورد بررسى قرار مى ميكيرد. وازدهاى كليدى: دادههاى آميخته طولى، آماسيدگى، اثرهاى تصادفى، مقادير گمشده. 


\section{| مقدمه}

يكى از مسائل مهم و رايج در مسائل علمى از قبيل روانشناسى، اقتصاد، سلامت و يزشكى وجود ياسخهاى

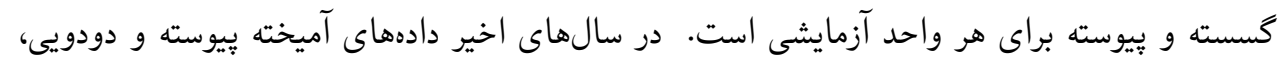

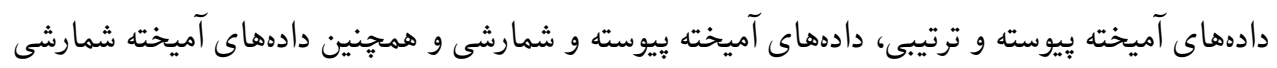

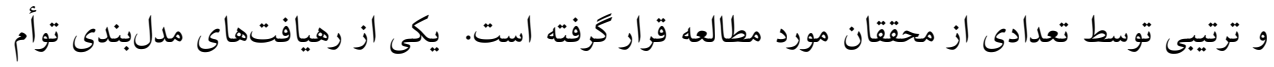

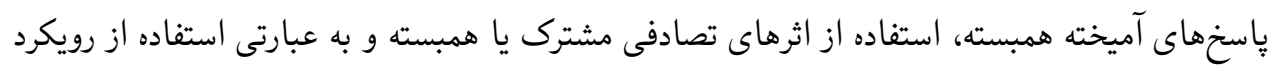

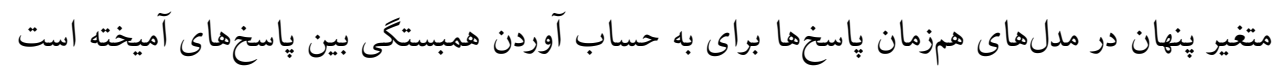

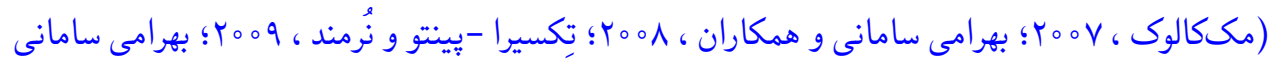

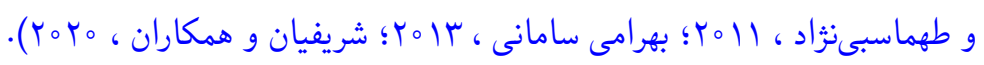

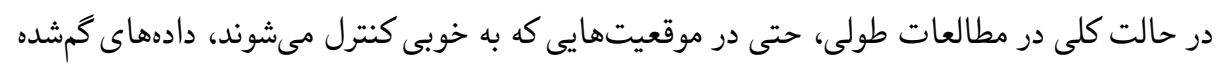

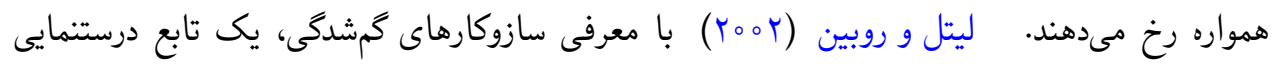

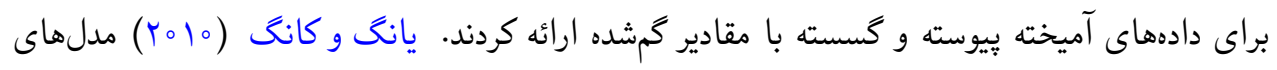

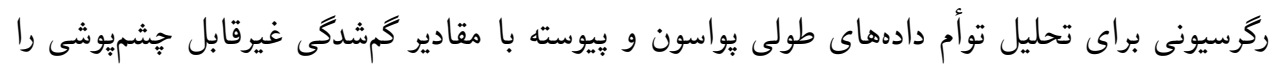

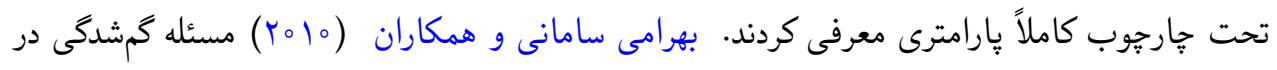

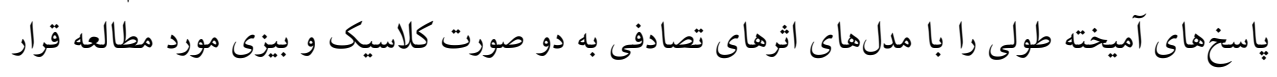

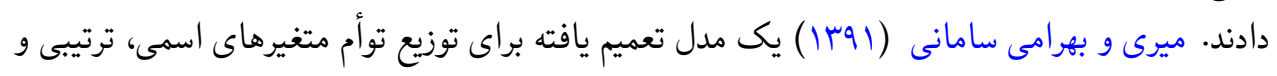

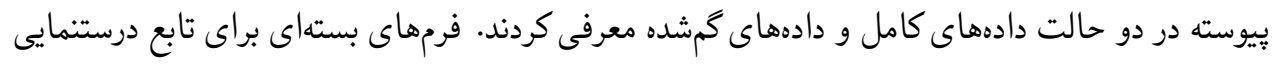

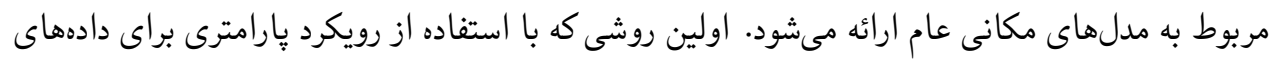

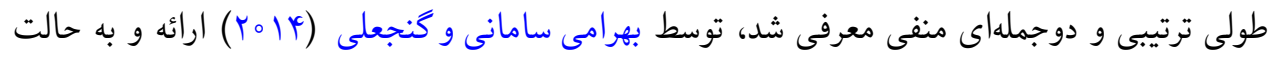

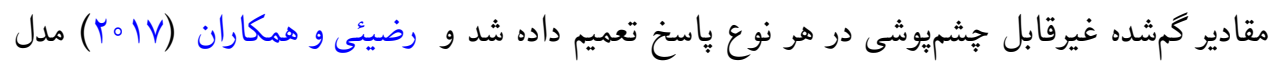

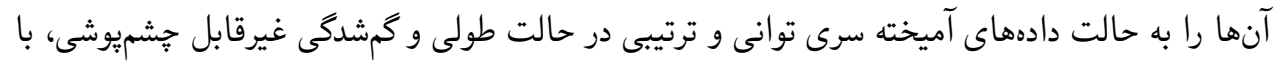
رويكرد متغير ينهان تعميم دادند.

مدل ركرسيون يواسون آماسيده در صفر براى دادهاى شمارشى شامل تعداد صفرهاى زياد، نخستين

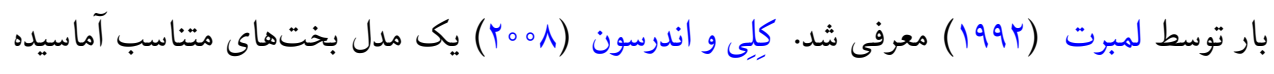

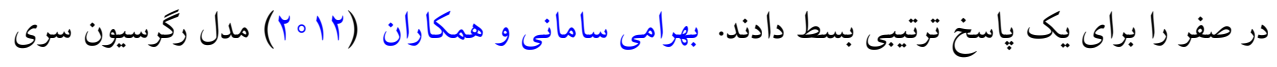

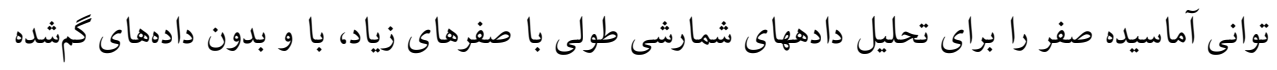

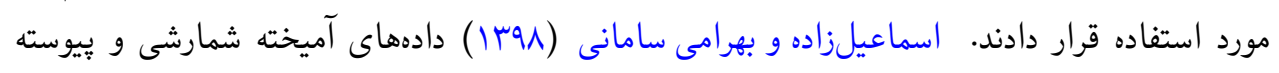


دومتغيرهاى را مورد تحليل قرار دادند كه متغير پاسخ شمارشى دومتغيره در يك يا دو نقطه آماسيده شده

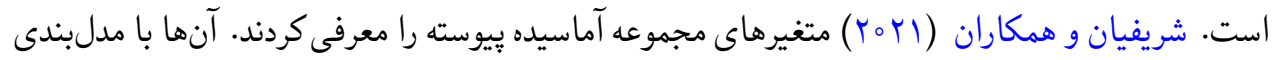

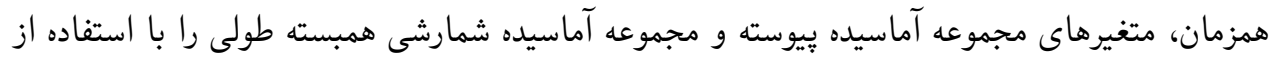

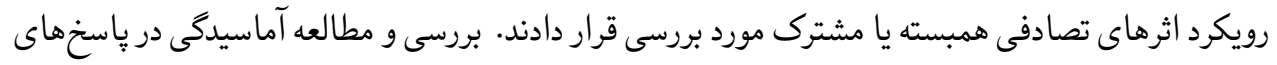

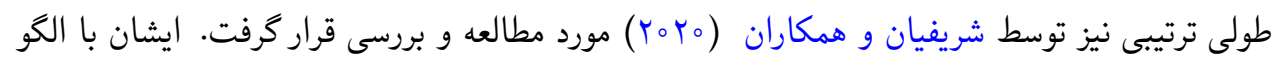

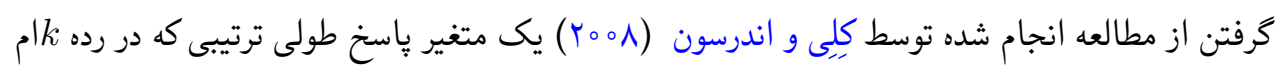

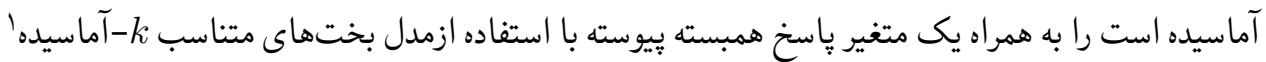
(k-IPO)

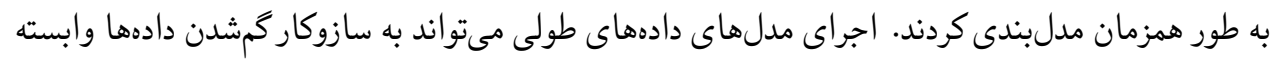

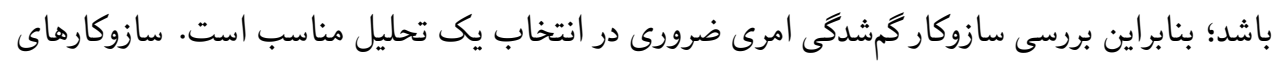

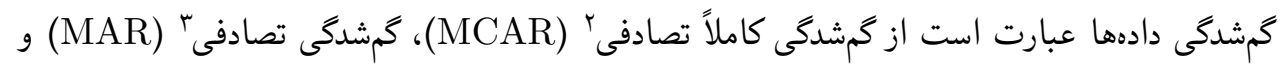

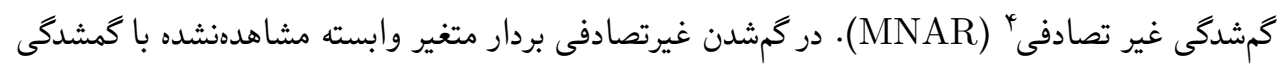

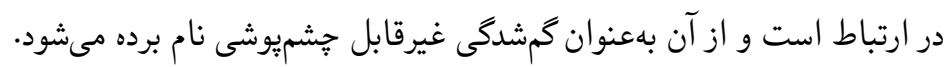
در اين مقاله به بررسى سازوكار گمشدگى غيرقابل جشميوشى در پاسخهاى آميخته مجموعه آماسيده

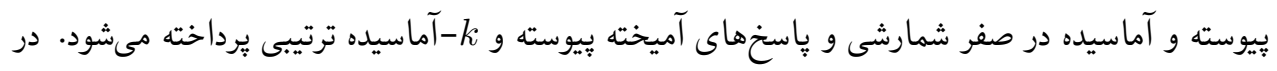

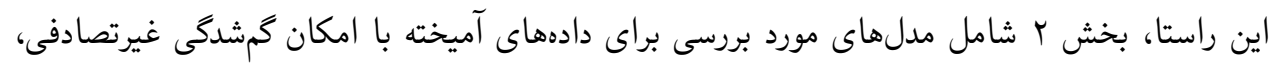

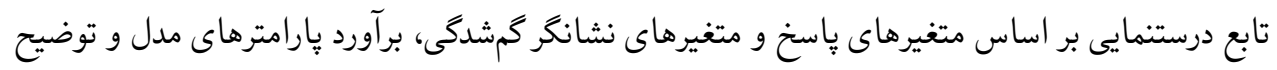

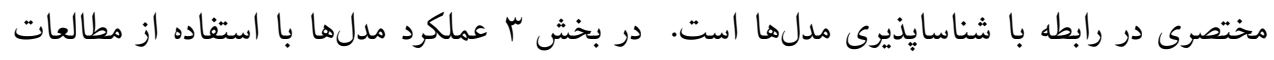

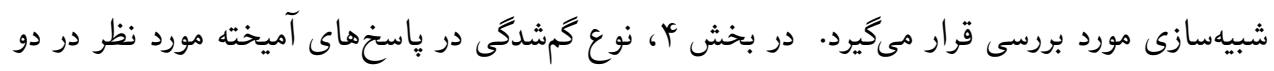

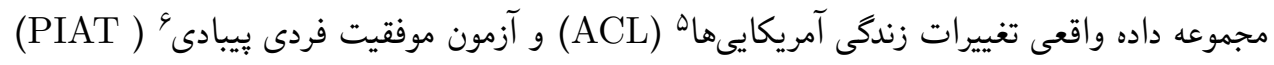

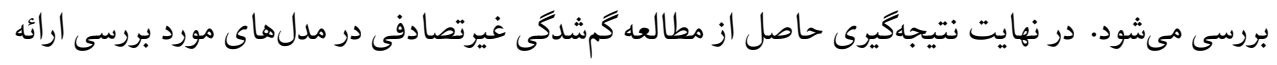
مىشود.

${ }^{1} \mathrm{k}$-Inflated Proportional odds ${ }^{2}$ Missing Completely at Random ${ }^{3}$ Missing at Random

${ }^{4}$ Missing Not at Random

5American's Changing Lives

${ }^{6}$ Peabody Individual Achievement Test 
كمشدكى غيرقابل جشميوشى در پِاسخهاى آميخته

\section{r ب r مدلها و درستنمايى}

در اين بخش امكان گمشدگى تصادفى در باسخهاى آميخته طولى مجموعه آماسيده بيوسته و آماسيده

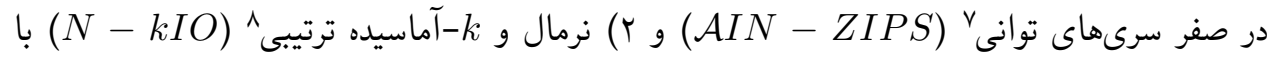

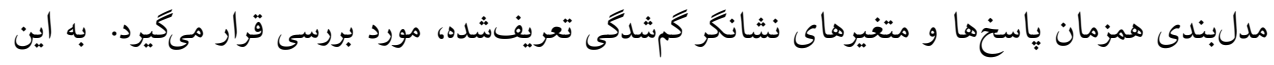

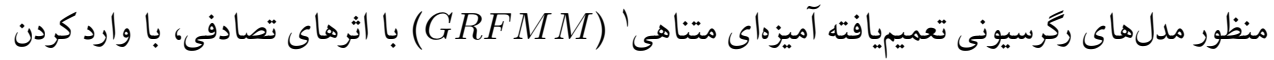

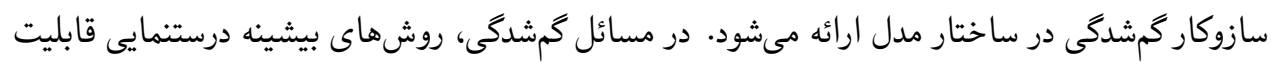

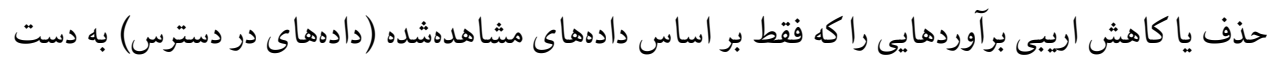

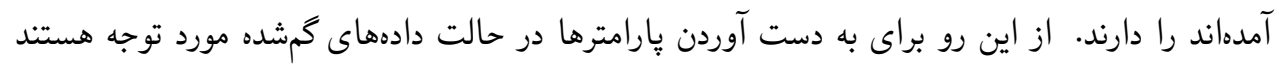

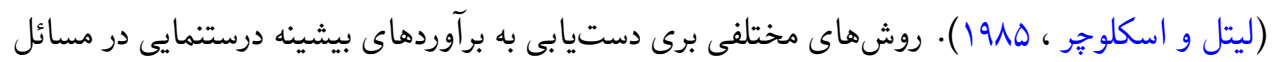

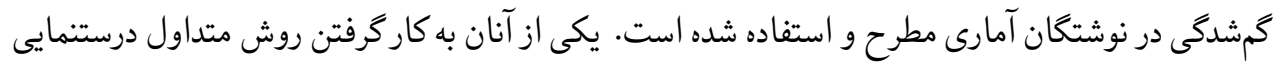

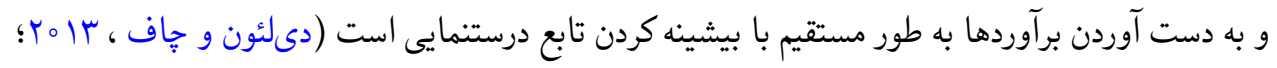

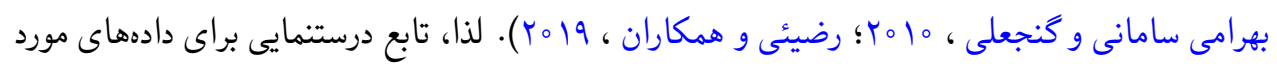

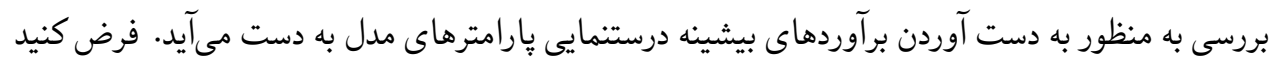
ي و و $Y_{i t}$

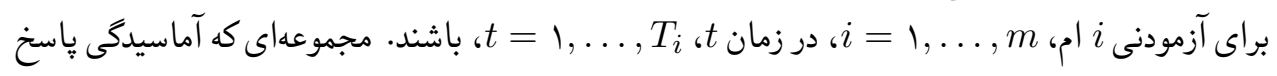

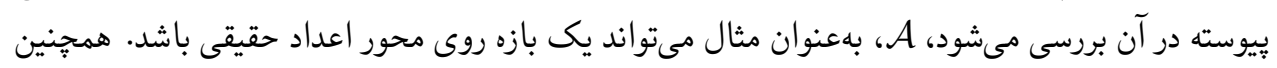
Yit

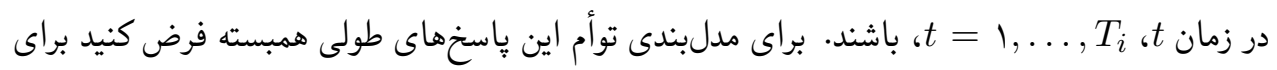

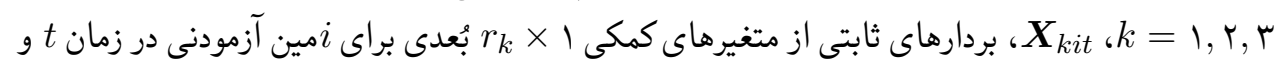
زئر $\boldsymbol{W}_{k i t}$

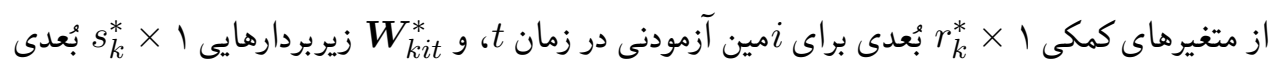
از أ باشند كه اكر متغيرهاى برابر با صفر هستند. باتشند

${ }^{7}$ A-Inflated Normal-Zero Inflated Power Series ${ }^{8}$ Normal-k Inflated Ordinal

${ }^{1}$ Generalized Regression Finite Mixture Model 


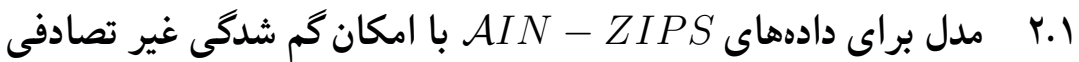

فرض مىشود كه

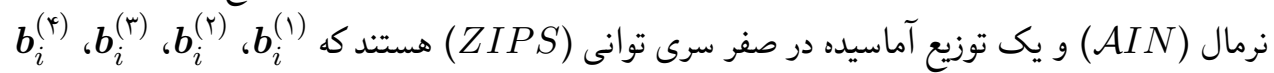
و

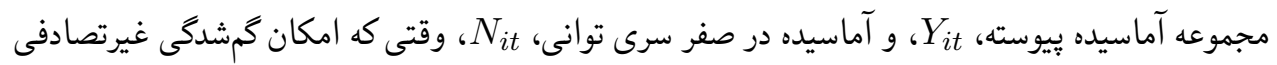

$$
\begin{aligned}
& Y_{i t} \mid \boldsymbol{b}_{i}^{(\backslash)}, \boldsymbol{b}_{i}^{(\curlyvee)}, \boldsymbol{b}_{i}^{(\Upsilon)}, \theta_{\mathcal{A}_{i t}}, \mu_{i t}, \pi_{\mathcal{A}_{i t}} \sim \mathcal{A} I N\left(\theta_{\mathcal{A}_{i t}}, \mu_{i t}, \sigma^{\curlyvee}, \sigma_{*}^{\curlyvee}, \pi_{\mathcal{A}_{i t}}\right), \\
& q_{\uparrow}\left(\mu_{i t}\right)=\boldsymbol{X}_{\backslash i t}^{\prime} \boldsymbol{\beta}_{t}^{(1)}+\boldsymbol{W}_{\backslash i t}^{\prime} \boldsymbol{b}_{i}^{(1)}, \\
& q_{\curlyvee}\left(\theta_{\mathcal{A}_{i t}}\right)=\boldsymbol{X}_{\curlyvee i t}^{\prime} \boldsymbol{\beta}_{t}^{(\Upsilon)}+\boldsymbol{W}_{\curlyvee i t}^{\prime} \boldsymbol{b}_{i}^{(\Upsilon)}, \\
& q_{\mathfrak{r}}\left(\pi_{\mathcal{A}_{i t}}\right)=\boldsymbol{X}_{\boldsymbol{r}_{i t}}^{\prime} \boldsymbol{\beta}_{t}^{(\boldsymbol{r})}+\boldsymbol{W}_{\boldsymbol{r}_{i t}}^{\prime} \boldsymbol{b}_{i}^{(\boldsymbol{r})}, \\
& \operatorname{logit}\left(\operatorname{Pr}\left(R_{y_{i t}}=|| \boldsymbol{M}_{\backslash i t}, y_{i t}\right)\right)=\boldsymbol{M}_{\backslash i t}^{\prime} \boldsymbol{\alpha}_{\imath t}^{(1)}+\alpha_{\uparrow t}^{(1)} y_{i t}, \\
& N_{i t} \mid \boldsymbol{b}_{i}^{(*)}, \boldsymbol{b}_{i}^{(\diamond)}, \nu_{i t}, \pi_{\circ i t} \sim \operatorname{ZIPS}\left(\nu_{i t}, \pi_{\circ i t}\right), \\
& q_{\uparrow}^{*}\left(\nu_{i t}\right)=\boldsymbol{X}_{i i t}^{*^{\prime}} \gamma_{t}^{(1)}+\boldsymbol{W}_{\backslash i t}^{*^{\prime}} \boldsymbol{b}_{i}^{(*)}, \\
& q_{\uparrow}^{*}\left(\pi_{\circ i t}\right)=\boldsymbol{X}_{\ulcorner i t}^{*^{\prime}} \boldsymbol{\gamma}_{t}^{(\uparrow)}+\boldsymbol{W}_{\curlyvee i t}^{*^{\prime}} \boldsymbol{b}_{i}^{(\diamond)}, \\
& \operatorname{logit}\left(\operatorname{Pr}\left(R_{n_{i t}}=1 \mid \boldsymbol{M}_{\curlyvee i t}, n_{i t}\right)\right)=\boldsymbol{M}_{\curlyvee i t}^{\prime} \boldsymbol{\alpha}_{\imath t}^{(\curlyvee)}+\alpha_{\curlyvee t}^{(\curlyvee)} n_{i t} .
\end{aligned}
$$

است، كه در آن

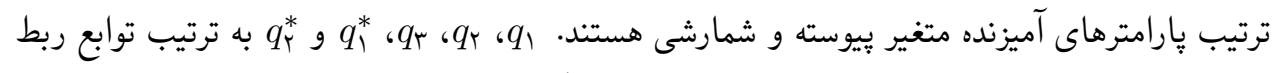

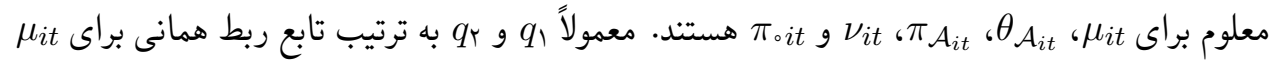
و تابع ربط لوجيت روى يك تبديل مكان-مقياس براى براى

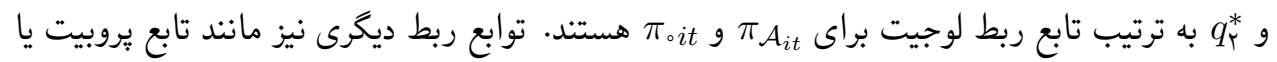

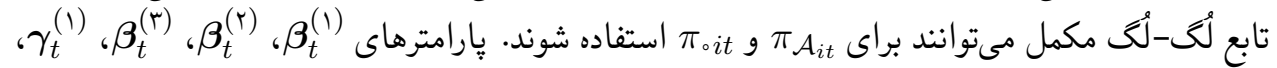

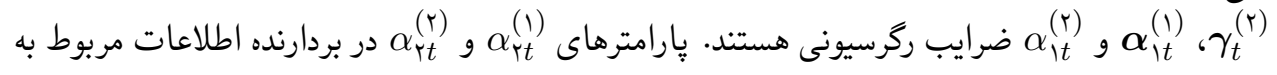

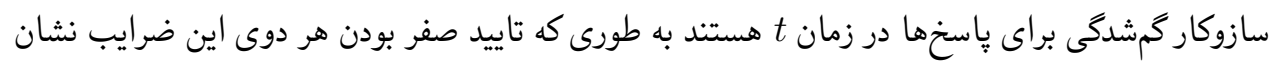

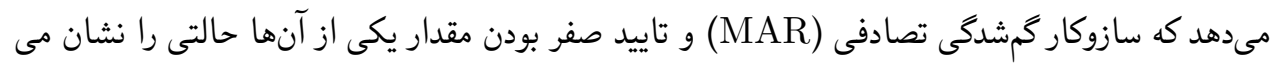




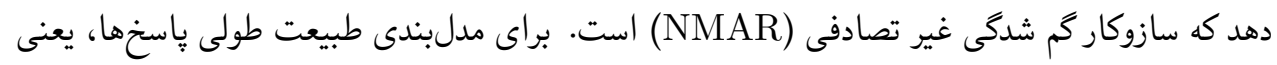

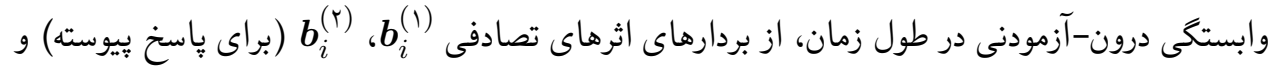
(ب)

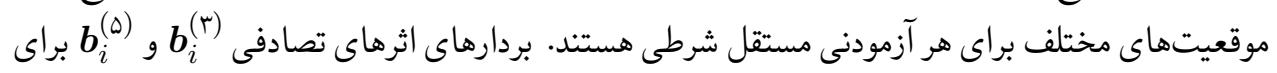

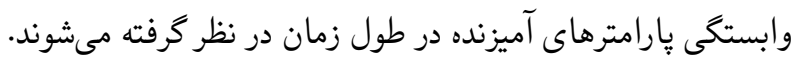

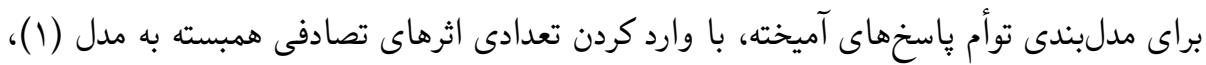
$\boldsymbol{v}_{i}^{(r)^{\prime}}=\boldsymbol{v}_{i}^{(1)^{\prime}}=\left(\boldsymbol{b}_{i}^{(1)^{\prime}}, \boldsymbol{b}_{i}^{(r)^{\prime}}, \boldsymbol{b}_{i}^{(r)^{\prime}}\right)$ همبستخ بين

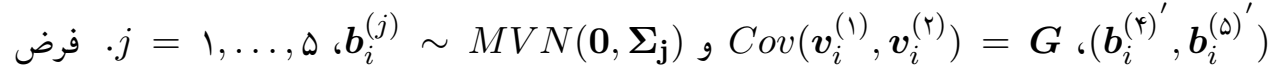

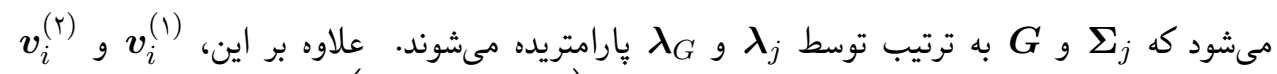
داراى توزيع كاوسى با ميانكين 0 و ماتريس كوواريانس $\Sigma_{v_{i}^{(\uparrow)}}=\left(\begin{array}{cc}\Sigma_{\psi} & 0 \\ 0 & \Sigma_{\triangleright}\end{array}\right), \Sigma_{v_{i}^{(1)}}=\left(\begin{array}{ccc}\Sigma_{1} & 0 & 0 \\ 0 & \Sigma_{\curlyvee} & 0 \\ 0 & 0 & \Sigma_{\digamma}\end{array}\right)$

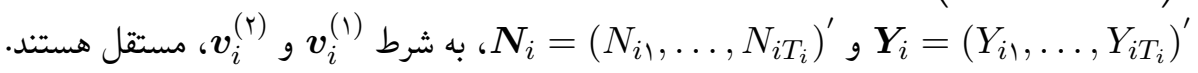

فرض كنيد

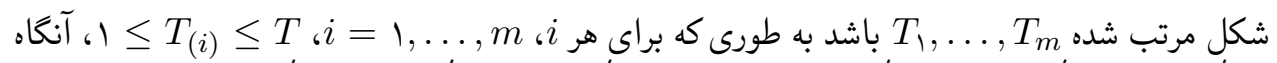
ر، كه $\boldsymbol{\gamma}^{(j)}=\left(\boldsymbol{\gamma}_{1}^{(j)^{\prime}}, \ldots, \boldsymbol{\gamma}_{T_{(1)}}^{(j)^{\prime}}, \ldots, \boldsymbol{\gamma}_{T_{(m)}}^{(j)^{\prime}}\right)^{\prime}, \boldsymbol{\beta}^{(j)}=\left(\boldsymbol{\beta}_{1}^{(j)^{\prime}}, \ldots, \boldsymbol{\beta}_{T_{(1)}}^{(j)^{\prime}}, \ldots, \boldsymbol{\beta}_{T_{(m)}}^{(j)^{\prime}}\right)^{\prime}$

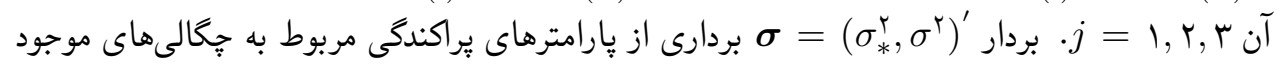
در مدل آميخته و و

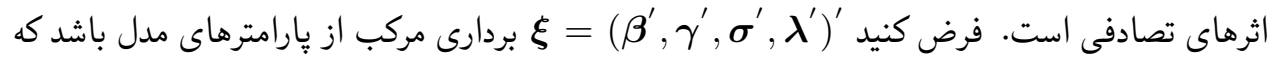
متعلق به فضاى بارامترى 


\section{تابع درستنمايى مدل (1)، حاصلضرب همه توابع درستنمايى تكى در نمونه به صورت}

$$
\begin{aligned}
& L(\boldsymbol{\xi})=\prod_{i=1}^{m} \int_{\mathcal{D}_{i}} \prod_{t=1}^{T_{i}} f\left(y_{i t}, n_{i t}, r_{y_{i t}}, r_{n_{i t}} \mid \boldsymbol{x}_{i t}, \boldsymbol{w}_{i t}, \boldsymbol{x}_{i t}^{*}, \boldsymbol{w}_{i t}^{*}, \boldsymbol{m}_{i t}, \boldsymbol{m}_{\curlyvee i t}, \boldsymbol{v}_{i}^{(\curlywedge)}, \boldsymbol{v}_{i}^{(\curlyvee)}\right) \\
& \times \psi\left(\boldsymbol{v}_{i}^{(1)}, \boldsymbol{v}_{i}^{(\Upsilon)}\right) d \boldsymbol{v}_{i}^{(1)} d \boldsymbol{v}_{i}^{(\Upsilon)} \\
& =\prod_{i=1}^{m} \int_{\mathcal{D}_{i}} \prod_{t=1}^{T_{i}} f\left(y_{i t} \mid \boldsymbol{x}_{i t}, \boldsymbol{w}_{i t}, \boldsymbol{v}_{i}^{(।)}, \boldsymbol{v}_{i}^{(ケ)}\right) \times f\left(n_{i t} \mid \boldsymbol{x}_{i t}^{*}, \boldsymbol{w}_{i t}^{*}, \boldsymbol{v}_{i}^{(।)}, \boldsymbol{v}_{i}^{(ケ)}\right) \\
& \times f\left(r_{y_{i t}} \mid \boldsymbol{m}_{\backslash i t}, y_{i t}\right) \times f\left(r_{n_{i t}} \mid \boldsymbol{m}_{\curlyvee i t}, n_{i t}\right) \times \psi\left(\boldsymbol{v}_{i}^{(!)}, \boldsymbol{v}_{i}^{(\vdash)}\right) d \boldsymbol{v}_{i}^{(।)} d \boldsymbol{v}_{i}^{(\digamma)} .
\end{aligned}
$$

است، كه در آن توزيع شىء تصادفى طور مشابه براى

$$
\begin{aligned}
& {\left[\pi_{\mathcal{A}_{i t}} g_{\mathcal{A}}\left(y_{i t} \mid \theta_{\mathcal{A}_{i t}}, \sigma_{*}^{r}\right)+\left(1-\pi_{\mathcal{A}_{i t}}\right) \phi\left(y_{i t} ; \mu_{i t}, \sigma^{r}\right)\right]^{z_{\mathcal{A}_{i t}}}} \\
& \times\left[\left(1-\pi_{\mathcal{A}_{i t}}\right) \phi\left(y_{i t} ; \mu_{i t}, \sigma^{r}\right)\right]^{\left(1-z_{\mathcal{A}_{i t}}\right)}, \\
& \text { و جگالى شرطى } \\
& {\left[\pi_{\circ i t}+\left(1-\pi_{\circ} \text { it }\right) \frac{a\left(n_{i t}\right) \nu_{i t}^{n_{i t}}}{g\left(\nu_{i t}\right)}\right]^{z_{\circ} i t} \times\left[\left(1-\pi_{\circ i t}\right) \frac{a\left(n_{i t}\right) \nu_{i t}^{n_{i t}}}{g\left(\nu_{i t}\right)}\right]^{\left(1-z_{\circ i t}\right)},}
\end{aligned}
$$

است، كه در آن آن يك توزيع دلخواه

$$
z_{\mathcal{A}_{i t}}=\left\{\begin{array}{cc}
1 & ; y_{i t} \in \mathcal{A} \\
\circ & ; y_{i t} \notin \mathcal{A}
\end{array} \quad, \quad z_{\text {oit }}=\left\{\begin{array}{cc}
1 & ; n_{i t}=。 \\
\circ & ; n_{i t} \neq \circ
\end{array}\right.\right.
$$




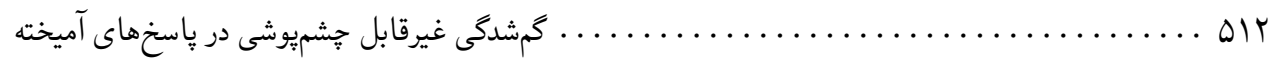

توزيع شرطى

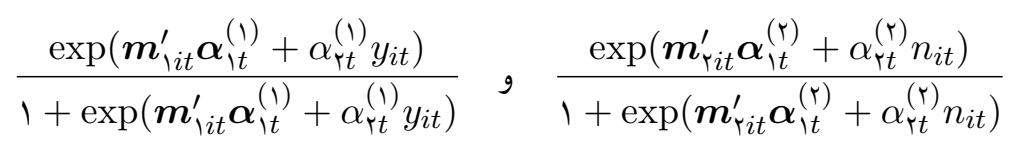

است. تابع درستنمايى (Y) در حالات مختلفى، بسته به اينكه كدام ياسخها در كدام زمانها شامل مقادير

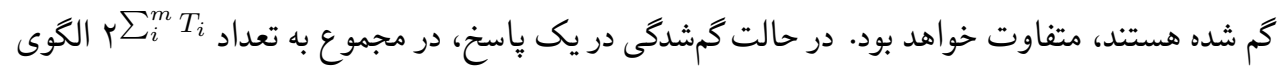

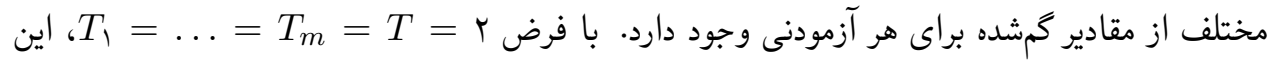

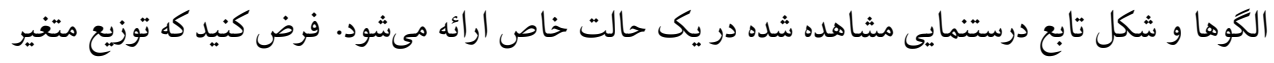

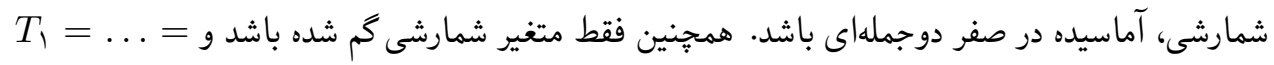
ان

$$
\text { اين حالت، تابع درستنمايى به صورت }
$$

$$
\begin{aligned}
& L(\boldsymbol{\xi})=\prod_{\left\{i: \boldsymbol{r}_{i}=1\right\}} f_{\boldsymbol{Y}_{i}, \boldsymbol{N}_{i}, \boldsymbol{R}_{i} \mid \boldsymbol{X}_{i}, \boldsymbol{M}_{i}}\left(\boldsymbol{y}_{i}, \boldsymbol{n}_{i}, \boldsymbol{r}_{i} \mid \boldsymbol{x}_{i}, \boldsymbol{m}_{i} ; \boldsymbol{\xi}\right) \\
& \times \prod_{\left\{i: r_{i}=\circ, r_{i \uparrow}=1\right\}} f_{\boldsymbol{Y}_{i}, \boldsymbol{N}_{i \curlyvee}, \boldsymbol{R}_{i} \mid \boldsymbol{X}_{i}, \boldsymbol{M}_{i}}\left(\boldsymbol{y}_{i}, \boldsymbol{n}_{i ץ}, \boldsymbol{r}_{i} \mid \boldsymbol{x}_{i}, \boldsymbol{m}_{i} ; \boldsymbol{\xi}\right) \\
& \times \prod_{\left\{i: r_{i \uparrow}=1, r_{i \uparrow}=\circ\right\}} f_{\boldsymbol{Y}_{i}, \boldsymbol{N}_{i}, \boldsymbol{R}_{i} \mid \boldsymbol{X}_{i}, \boldsymbol{M}_{i}}\left(\boldsymbol{y}_{i}, \boldsymbol{n}_{i \uparrow}, \boldsymbol{r}_{i} \mid \boldsymbol{x}_{i}, \boldsymbol{m}_{i} ; \boldsymbol{\xi}\right) \\
& \times \prod_{\left\{i: r_{i} \mid=\circ, r_{i \uparrow}=\circ\right\}} f_{\boldsymbol{Y}_{i}, \boldsymbol{R}_{i} \mid \boldsymbol{X}_{i}, \boldsymbol{M}_{i}}\left(\boldsymbol{y}_{i}, \boldsymbol{r}_{i} \mid \boldsymbol{x}_{i}, \boldsymbol{m}_{i} ; \boldsymbol{\xi}\right) \\
& =\prod_{i=1}^{m}\left[f\left(y_{i}, n_{i \uparrow}, n_{i \curlyvee} ; \boldsymbol{\xi}\right)\right]^{r_{i \uparrow} r_{i \curlyvee}}\left[f\left(y_{i}, N A, n_{i \uparrow} ; \boldsymbol{\xi}\right)\right]^{\left(1-r_{i \uparrow}\right) r_{i \curlyvee}} \\
& \times\left[f\left(y_{i}, n_{i \uparrow}, N A ; \boldsymbol{\xi}\right)\right]^{r_{i 1}\left(1-r_{i \uparrow}\right)}\left[f\left(y_{i}, N A, N A ; \boldsymbol{\xi}\right)\right]^{\left(1-r_{i \uparrow}\right)\left(1-r_{i \uparrow}\right)},
\end{aligned}
$$


DIT نسترن شريفيان، احسان بهرامى سامانى .

$$
\begin{aligned}
& \text { است، كه در آن R متغير نشانغر مربوط به گمشدن متغير شمارشى است و }
\end{aligned}
$$

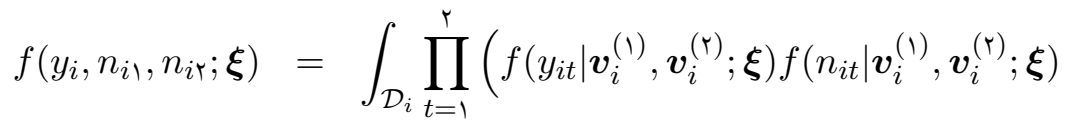

$$
\begin{aligned}
& \left.\times \operatorname{Pr}\left(R_{i t}=1 \mid y_{i t} ; \boldsymbol{\xi}\right)\right) \psi\left(\boldsymbol{v}_{i}^{(1)}, \boldsymbol{v}_{i}^{(\digamma)}\right) d \boldsymbol{v}_{i}^{(1)} d \boldsymbol{v}_{i}^{(\digamma)}, \\
& f\left(y_{i}, N A, n_{i \Upsilon} ; \boldsymbol{\xi}\right)=\int_{\mathcal{D}_{i}}\left(\prod_{t=1}^{r} f\left(y_{i t} \mid \boldsymbol{v}_{i}^{(।)}, \boldsymbol{v}_{i}^{(\Upsilon)} ; \boldsymbol{\xi}\right)\right) \operatorname{Pr}\left(R_{i \uparrow}=\circ \mid \boldsymbol{\xi}\right) \\
& \times \quad f\left(n_{i \digamma} \mid \boldsymbol{v}_{i}^{(1)}, \boldsymbol{v}_{i}^{(\digamma)} ; \boldsymbol{\xi}\right) \psi\left(\boldsymbol{v}_{i}^{(।)}, \boldsymbol{v}_{i}^{(\digamma)}\right) d \boldsymbol{v}_{i}^{(1)} d \boldsymbol{v}_{i}^{(\digamma)}, \\
& f\left(y_{i}, N A, N A ; \boldsymbol{\xi}\right)=\int_{\mathcal{D}_{i}}\left(\prod_{t=1}^{\uparrow} f\left(y_{i t} \mid \boldsymbol{v}_{i}^{(!)}, \boldsymbol{v}_{i}^{(\Upsilon)} ; \boldsymbol{\xi}\right)\right) \operatorname{Pr}\left(R_{i \curlyvee}=\circ \mid \boldsymbol{\xi}\right) \\
& \times \operatorname{Pr}\left(R_{i \backslash}=\circ \mid \boldsymbol{\xi}\right) \psi\left(\boldsymbol{v}_{i}^{(\perp)}, \boldsymbol{v}_{i}^{(\digamma)}\right) d \boldsymbol{v}_{i}^{(\perp)} d \boldsymbol{v}_{i}^{(\digamma)} .
\end{aligned}
$$

$f\left(y_{i}, n_{i \uparrow}, N A ; \boldsymbol{\xi}\right)$ آماسيده در صفر دوجملهاى $n$ و

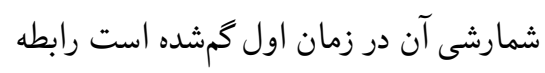

$$
\begin{aligned}
\operatorname{Pr}\left(R_{i \uparrow}=\circ \mid \boldsymbol{\xi}\right) & =\sum_{n_{i \uparrow}=\circ}^{n}\left(1-\operatorname{Pr}\left(R_{i \uparrow}=|| n_{i \uparrow} ; \boldsymbol{\xi}\right)\right)\left(\left(\pi_{i \uparrow}+\frac{1-\pi_{i \backslash}}{\left(1+\nu_{i \backslash}\right)^{n}}\right)^{z_{\circ i}}\right. \\
& \left.\times\left(\left(1-\pi_{i \uparrow}\right)\left(\begin{array}{c}
n \\
n_{i \uparrow}
\end{array}\right)\left(\frac{\nu_{i \backslash}}{1+\nu_{i \uparrow}}\right)^{n_{i \backslash}}\left(\frac{1}{1+\nu_{i t}}\right)^{n-n_{i \backslash}}\right)^{1-z_{i t}}\right) .
\end{aligned}
$$

برقرار است. به همين شكل در حالتهاى مختلف اين احتمالها قابل محاسبه است. موارد ديخر نيز به شكل مشابه به دست مى آيند.

انتكرال موجود در رابطه (Y) كه نسبت به بردارهاى اثرهاى تصادفى است با روش مونت كارلو تقريب

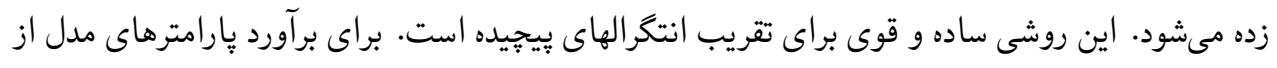

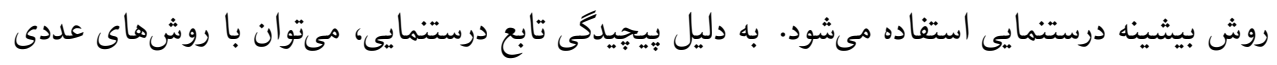

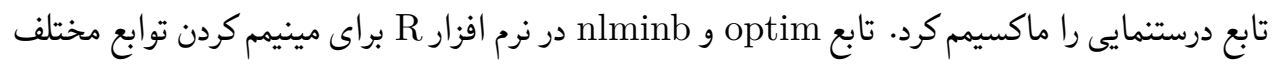

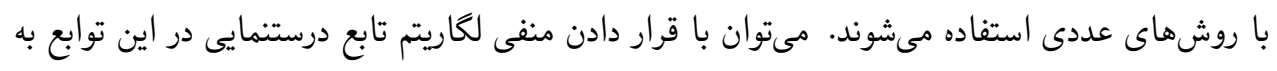

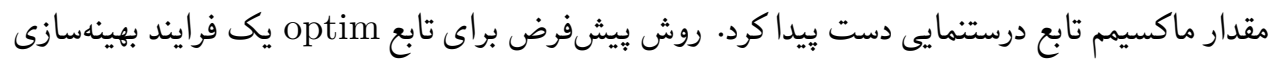


كَشدى غيرقابل جشميوشى در باسخهاى آميخته

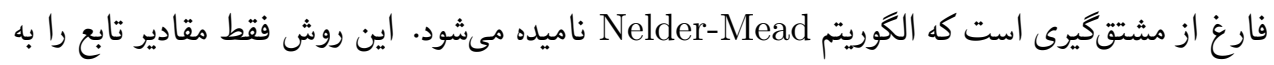
كار مىبرد، استوار اما نسبتاً كند است و به طور منطقى براى توابع مشتق نايذير خوب عمل ميكند.

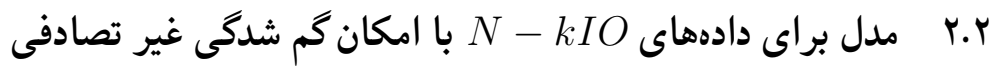

فرض مىشود كه جندجملهاى (kIMULT) هستند كه

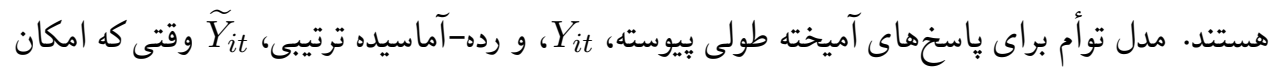
كمشدكى غيرتصادفى وجود دارد به صورت

$$
\begin{aligned}
& Y_{i t} \mid \boldsymbol{b}_{i}^{(1)}, \mu_{i t} \sim N\left(\mu_{i t}, \sigma^{\lceil} \backslash\right), \\
& q_{\curlywedge}\left(\mu_{i t}\right)=\boldsymbol{X}_{\backslash i t}^{\prime} \boldsymbol{\beta}_{t}^{(1)}+\boldsymbol{W}_{\uparrow i t}^{\prime} \boldsymbol{b}_{i}^{(1)}, \\
& \operatorname{logit}\left(\operatorname{Pr}\left(R_{y_{i t}}=|| \boldsymbol{M}_{\backslash i t}, y_{i t}\right)\right)=\boldsymbol{M}_{\backslash i t}^{\prime} \boldsymbol{\alpha}_{\downarrow t}^{(1)}+\alpha_{\curlyvee t}^{(1)} y_{i t}, \\
& \widetilde{Y}_{i t} \mid \boldsymbol{b}_{i}^{(\Upsilon)}, \boldsymbol{b}_{i}^{(\digamma)}, \eta_{j, i t}, \pi_{i t} \sim k \operatorname{IMULT}\left(\backslash, \eta_{\circ}, i t, \ldots, \eta_{c-1, i t}, \pi_{i t}\right), \\
& \operatorname{logit}\left(\eta_{j, i t}\right)=\theta_{j}-\left(\boldsymbol{X}_{\curlyvee i t}^{\prime} \boldsymbol{\gamma}^{(1)}+\boldsymbol{W}_{\curlyvee i t}^{\prime} \boldsymbol{b}_{i}^{(\uparrow)}\right), j=\circ, 1, \ldots, c-1 \\
& q_{\uparrow}\left(\pi_{i t}\right)=\boldsymbol{X}_{\boldsymbol{r}_{i t}}^{\prime} \boldsymbol{\gamma}^{(\uparrow)}+\boldsymbol{W}_{\boldsymbol{r} i t}^{\prime} \boldsymbol{b}_{i}^{(\boldsymbol{r})} \\
& \operatorname{logit}\left(\operatorname{Pr}\left(R_{\widetilde{Y}_{i t}}=|| \boldsymbol{M}_{\curlyvee i t}, \widetilde{y}_{i t}\right)\right)=\boldsymbol{M}_{\curlyvee i t}^{\prime} \boldsymbol{\alpha}_{\uparrow t}^{(\curlyvee)}+\alpha_{\curlyvee t}^{(\uparrow)} \widetilde{y}_{i t} .
\end{aligned}
$$

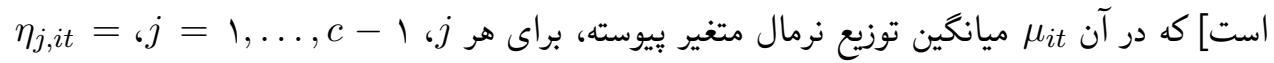

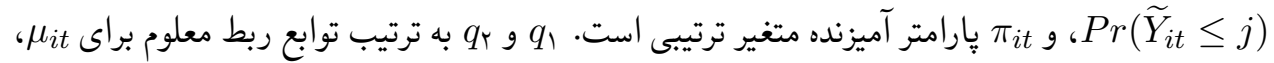

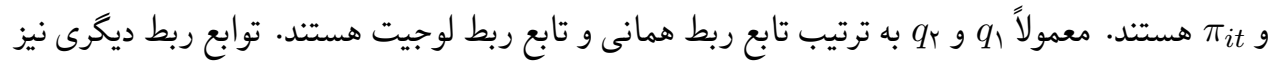

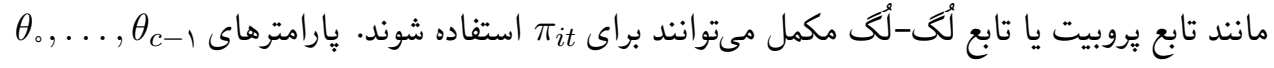

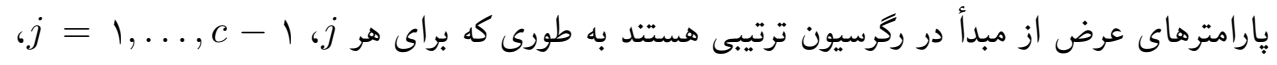
بارئ تايد

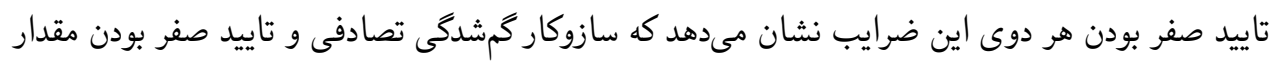

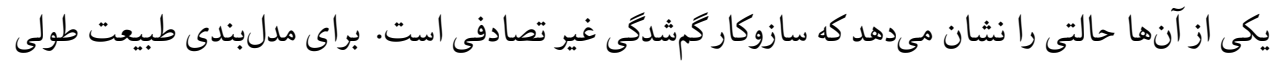

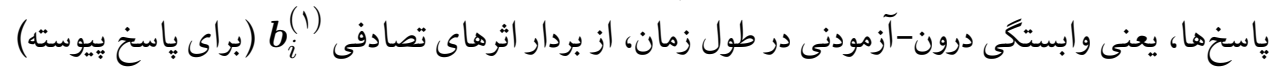


و (؟)

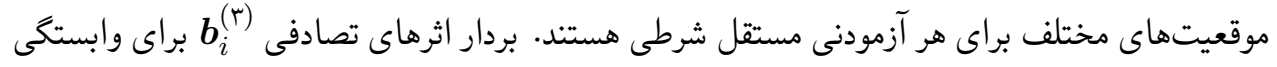

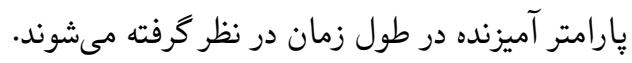

هماند مدل (1) براى مدلبندى توأم ياسخهاى آميخته، با وارد كردن اثرهاى تصادفى همبسته به

مدل (Y) ما همبستخى بين ماتريس واريانس-كوواريانس بردار عاون

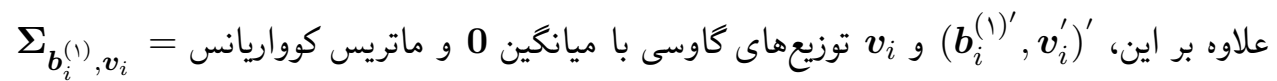

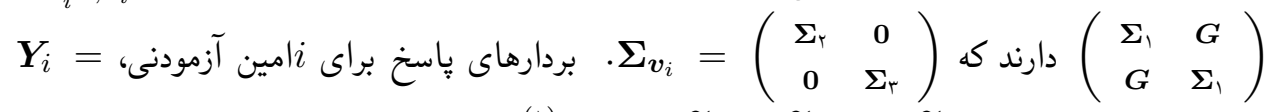

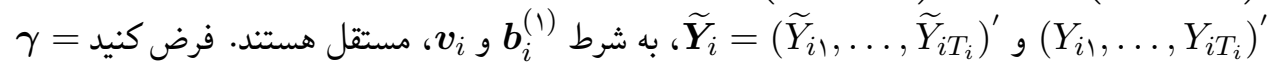
(I) $\boldsymbol{\gamma}^{(j)}=\boldsymbol{\beta}^{(1)}=\left(\boldsymbol{\beta}_{1}^{(1)^{\prime}}, \ldots, \boldsymbol{\beta}_{T_{(1)}^{(1)}}^{\prime}, \ldots, \boldsymbol{\beta}_{T_{(m)}^{(j)^{\prime}}}^{\prime}\right)^{\prime}$ 。

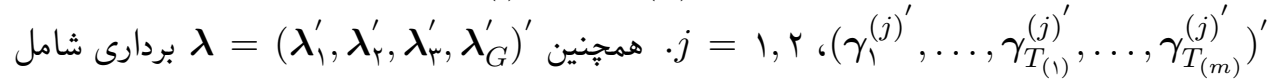
$\boldsymbol{\xi}=\left(\boldsymbol{\beta}^{\prime}, \boldsymbol{\gamma}^{\prime}, \boldsymbol{\lambda}^{\prime}, \sigma^{r}\right)^{\prime}{ }^{\prime}$ بردارى مركب از پارامترهاى مدل باشد كه متعلق به كه d تعداد همه پِارامترهاى مدل است). تابع درستنمايى مدل (†)، حاصلضرب همه توابع درستنمايى تكى در نمونه به صورت

$$
\begin{aligned}
& L(\boldsymbol{\xi})=\prod_{i=1}^{m} \int_{\mathcal{D}_{i}} \prod_{t=1}^{T_{i}} f\left(y_{i t}, \widetilde{y}_{i t}, r_{y_{i t}}, r_{\widetilde{y}_{i t}} \mid \boldsymbol{x}_{i t}, \boldsymbol{w}_{i t}, \boldsymbol{m}_{\backslash i t}, \boldsymbol{m}_{\curlyvee i t}, \boldsymbol{b}_{i}^{(\backslash)}, \boldsymbol{v}_{i}\right) \\
& \times \psi\left(\boldsymbol{b}_{i}^{(!)}, \boldsymbol{v}_{i}\right) d \boldsymbol{b}_{i}^{(1)} d \boldsymbol{v}_{i} \\
& =\prod_{i=1}^{m} \int_{\mathcal{D}_{i}} \prod_{t=1}^{T_{i}} f\left(y_{i t} \mid \boldsymbol{x}_{\backslash i t}, \boldsymbol{w}_{\backslash i t}, \boldsymbol{b}_{i}^{(।)}, \boldsymbol{v}_{i}\right) \times f\left(\widetilde{y}_{i t} \mid \boldsymbol{x}_{\curlyvee i t}, \boldsymbol{w}_{\curlyvee i t}, \boldsymbol{x}_{\curlyvee i t}, \boldsymbol{w}_{\curlyvee i t}, \boldsymbol{b}_{i}^{(\backslash)}, \boldsymbol{v}_{i}\right) \\
& \times f\left(r_{y_{i t}} \mid \boldsymbol{m}_{\backslash i t}, y_{i t}\right) \times f\left(r_{\widetilde{y}_{i t}} \mid \boldsymbol{m}_{\curlyvee i t}, \widetilde{y}_{i t}\right) \times \psi\left(\boldsymbol{b}_{i}^{(।)}, \boldsymbol{v}_{i}\right) d \boldsymbol{b}_{i}^{(1)} d \boldsymbol{v}_{i}
\end{aligned}
$$

است، كه در آن 
شى تصادفى براى

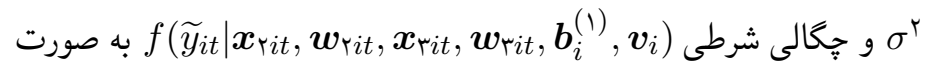

$$
\begin{aligned}
{\left[\pi_{i t}+\left(1-\pi_{i t}\right)\left(\eta_{k, i t}\right.\right.} & \left.\left.-\eta_{k-1, i t}\right)\right]^{z_{i t}} \\
& \times\left[\left(1-\pi_{i t}\right) \sum_{j \in\{0, \ldots, c\}-k}\left[\left(\eta_{j, i t}-\eta_{j-1, i t}\right) I_{\{j\}}\left(\widetilde{y}_{i t}\right)\right]\right]^{1-z_{i t}}
\end{aligned}
$$

است، كه در آن وقتى

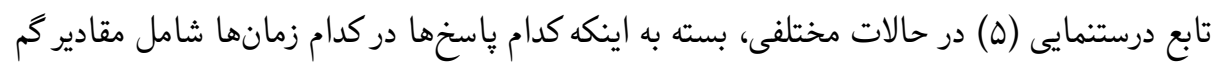

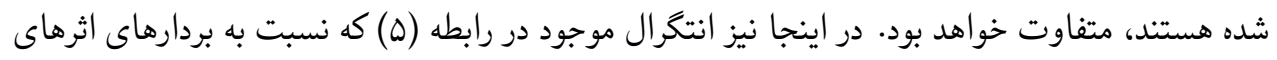

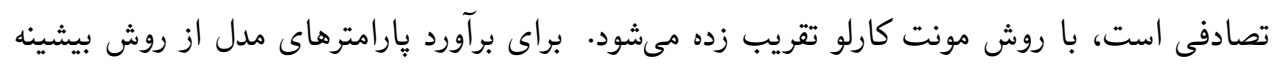

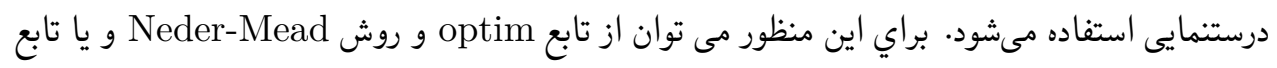
nlminb

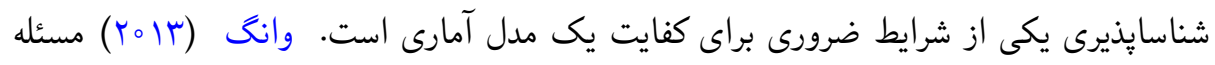

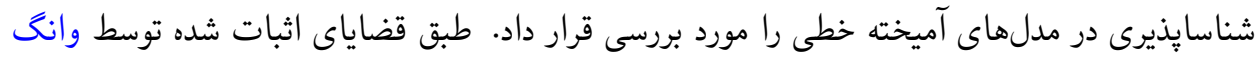

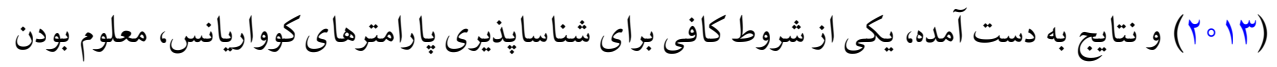

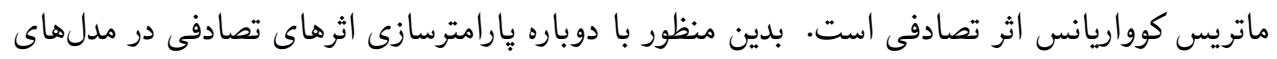
مورد بررسى به مسئله شناسايذيرى توجه شده است.

\section{r م م مالعات شبيهسازى}

در اين بخش عملكرد مدل (1) و (†) با تعدادى مطالعه شبيهازى مورد ارزيابى قرار مىگيرد. نمونهاى شبيهسازى با جهار اندازه نمونه

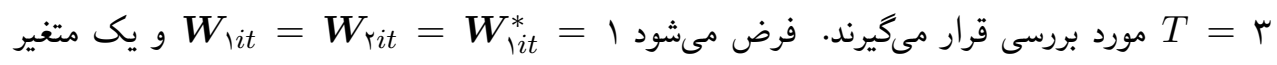
زمان-متغير \ا

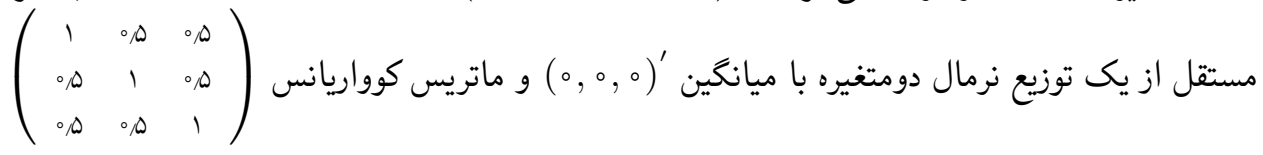




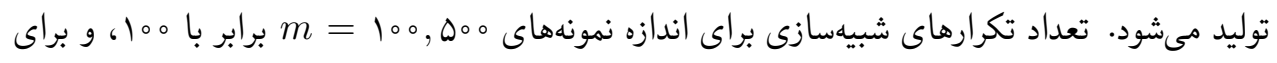
اندازه نمونههاى

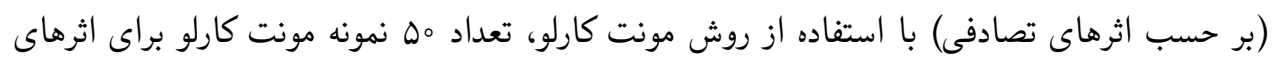

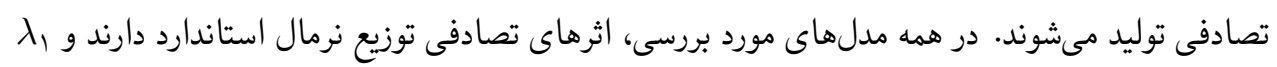

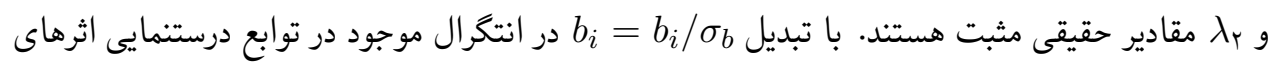
تصادفى بازمقياس مىشوند و به جاى هر اثر تصادفى همجِون

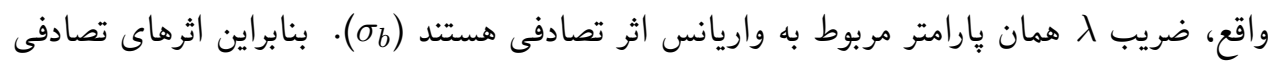

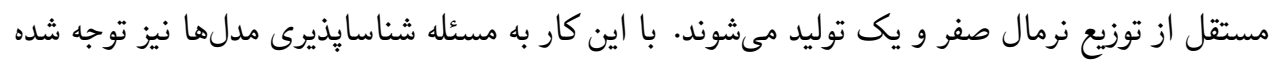

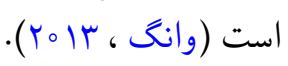

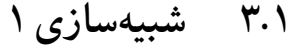

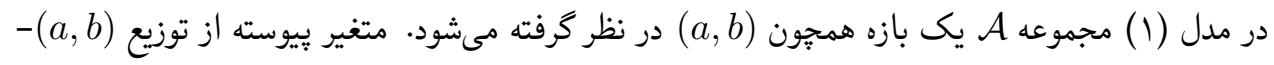

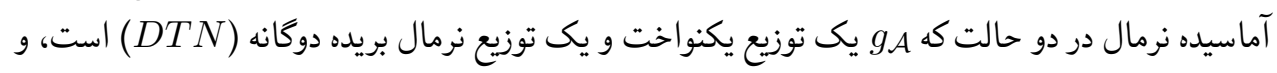

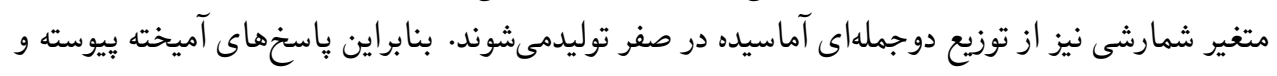

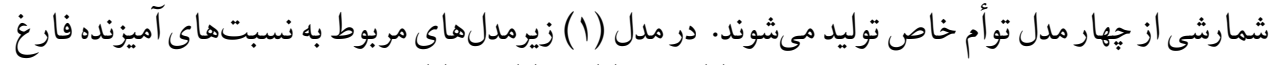
از اثر تصادفى خواهند بود و فرض مىشود كه آز

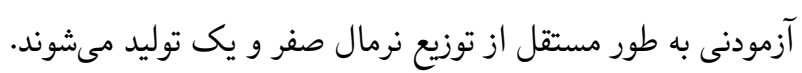

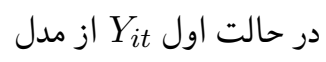

$$
\begin{aligned}
& Y_{i t} \mid b_{i}^{(\backslash)}, \mu_{i t}, \pi_{\mathcal{A}_{i t}}, \sigma^{\curlyvee} \sim(a, b) I N\left(\mu_{i t}, \pi_{\mathcal{A}_{i t}}, \sigma^{\curlyvee}\right) ; \quad g_{\mathcal{A}_{i t}} \sim U(a, b) \\
& \mu_{i t}=\beta_{\circ}^{(1)}+\beta_{1}^{(1)} X_{1 i t}+\lambda_{1} b_{i}^{(1)} \text {, } \\
& \operatorname{logit}\left(\pi_{\mathcal{A}_{i t}}\right)=\beta_{\circ}^{(\Gamma)}+\beta_{1}^{(\Gamma)} X_{\backslash i t},
\end{aligned}
$$


1

و

$$
\begin{aligned}
& N_{i t} \mid b_{i}^{(1)}, \nu_{i t}, \pi_{\circ} i t \sim Z I B\left(\vee, \frac{\nu_{i t}}{1+\nu_{i t}}, \pi_{\circ i t}\right), \\
& \log \left(\nu_{i t}\right)=\gamma_{0}^{(1)}+\gamma_{1}^{(1)} X_{\backslash i t}+\lambda_{1} b_{i}^{(1)}, \\
& \operatorname{logit}\left(\pi_{\circ i t}\right)=\gamma_{\circ}^{(\Upsilon)}+\gamma_{\uparrow}^{(\Upsilon)} X_{\backslash i t} .
\end{aligned}
$$

توليد مىشود. در حالت دوم

$Y_{i t} \mid b_{i}^{(।)}, b_{i}^{(\Upsilon)}, \theta_{i t}^{*}, \mu_{i t}, \pi_{\mathcal{A}_{i t}}, \sigma_{*}^{\curlyvee}, \sigma^{\curlyvee} \sim(a, b) I N\left(\theta_{i t}^{*}, \mu_{i t}, \pi_{\mathcal{A}_{i t}}, \sigma_{*}^{\curlyvee}, \sigma^{\Upsilon}\right) ; g_{\mathcal{A}_{i t}} \sim \operatorname{DTN}_{(a, b)}$

$\mu_{i t}=\beta_{\circ}^{(1)}+\beta_{\backslash t}^{(1)} X_{\backslash i t}+\lambda_{1} b_{i}^{(1)}$,

$\operatorname{logit}\left(\theta_{i t}^{*}\right)=\beta_{\circ}^{(\Upsilon)}+\beta_{\backslash t}^{(\Upsilon)} X_{\backslash i t}+\lambda_{\uparrow} b_{i}^{(\Upsilon)}$,

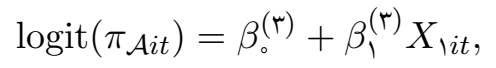

و $N_{i t}$

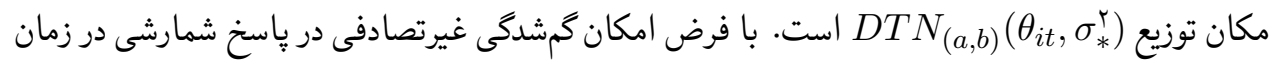

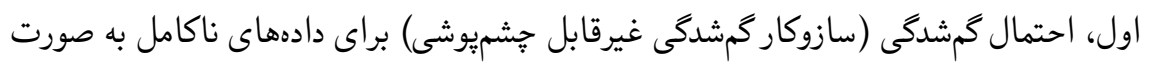

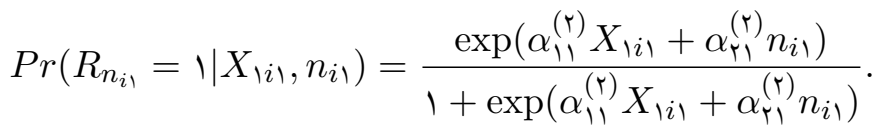

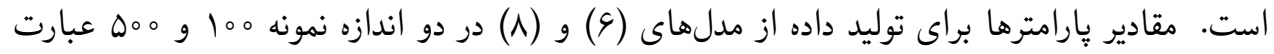
$\left(\gamma_{0}^{(1)}, \gamma_{1}^{(1)}, \gamma_{0}^{(r)}, \gamma_{1}^{(r)}\right)=$ ، ( $\left.\beta_{0}^{(1)}, \beta_{1}^{(1)}, \beta_{0}^{(r)}, \beta_{1}^{(r)}, \beta_{0}^{(r)}, \beta_{1}^{(r)}\right)=(0,1,1,-0,1,1,1,1)$

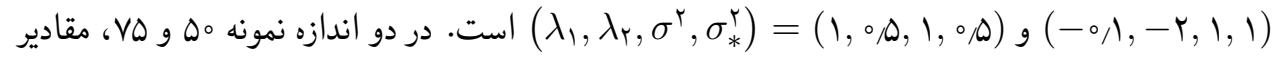
واقعى پارامترهاى مدل

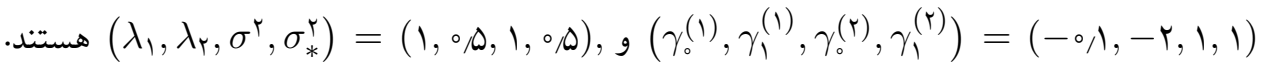

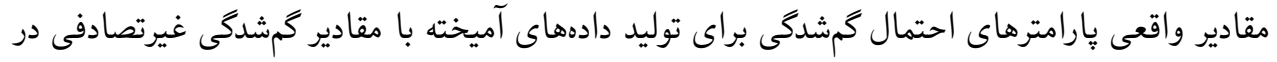

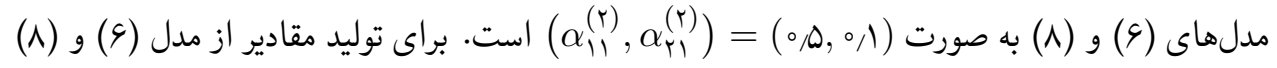

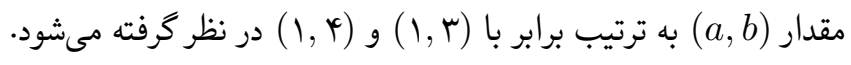




\begin{tabular}{|c|c|c|c|c|c|c|c|c|c|}
\hline \multicolumn{4}{|c|}{$m=\Delta^{\circ \circ}$} & \multicolumn{4}{|c|}{$m=100$} & \multirow[b]{3}{*}{ مقدار واقعى } & \multirow[b]{3}{*}{ يَارامتر } \\
\hline \multicolumn{2}{|c|}{ (ب) } & \multicolumn{2}{|c|}{ (الف) } & \multicolumn{2}{|c|}{ (ب) } & \multicolumn{2}{|c|}{ (الف) } & & \\
\hline $\mathrm{SE}$ & برآورد & $\mathrm{SE}$ & برآورد & $\mathrm{SE}$ & برآورد & $\mathrm{SE}$ & برآورد & & \\
\hline$\circ / 10$ & $\circ \lambda \wedge$ & 0,090 & $0 / 194$ & $\circ \pi \wedge 9$ & 0, TIF & $\circ+4 \Delta \circ$ & $\circ$ r.t. & $0>$ & $\beta_{0}^{(1)}$ \\
\hline$\circ, \wedge \Lambda$ & -AVG & $\circ \% \mathrm{VG}$ & ०/AG & 0,199 & $1 / 11$ & 0,419 & $M \circ \Lambda$ & $1 / 0$ & $\beta_{1}^{(1)}$ \\
\hline 0,091 & $M \circ 4$ & $0,0 \mathrm{VT}$ & $1, \circ \mu \wedge$ & $0, r_{01}$ & $0 / \wedge 01$ & 0,190 & o & $1 / 0$ & $\lambda_{1}$ \\
\hline $0, \pi 10$ & 1,ory & $0 / 0 \Delta \Lambda$ & 1,o०r & $0, x_{01}$ & سחזו & $0 / 1 V$ & 0,900 & $1 / 0$ & $\sigma^{r}$ \\
\hline $0 / 111$ & - QAT & $\circ \lambda \circ 0$ & 0,900 & $0 / 194$ & MTY & $0, Y \wedge 1$ & M & $1 / 0$ & $\beta_{0}^{(r)}$ \\
\hline $0 / 104$ & $1 / 019$ & $0 / 1 V$ & 1,079 & 0,191 & $|\pi| V$ & $0, Y Y A$ & $1, r \circ \Delta$ & $1 / 0$ & $\beta_{!}^{(r)}$ \\
\hline$\circ / \wedge r$ & $-0,091$ & 0,091 & - ו ו & ०ग149 & שrונים & $0 / 19 V$ & $-0 / 10$ & $-0 / 1$ & \\
\hline$\circ \% \mathrm{VD}$ & $-r / 1 \circ 1$ & $\circ /{ }^{\circ} \Lambda$ & $-r_{/} r V$ & O & $-\mid N \psi_{0}$ & $0, Y \wedge G$ & $-|/ 9 \wedge|$ & $-r_{/ 0}$ & \\
\hline $0,09 V$ & ONTI & $0 / 0 \wedge 9$ & $0,99 \wedge$ & 0,491 & 0,919 & $0,4 \circ r$ & $\% \Delta \Delta r$ & $1 / 0$ & \\
\hline $0, Y \circ Y$ & $1 / 091$ & $0>109$ & $1, r \circ r$ & $0, \pi r q$ & $1 / 101$ & ofry & $1 / 1 \circ \Lambda$ & $1 / 0$ & \\
\hline $0, x \circ 1$ & $1,0 \wedge t$ & 0 XIV & $1,09 V$ & $0,49 V$ & IMYV & $0,4 V$ & $|x+4|$ & $1 / 0$ & $\alpha_{i !}^{(r)}$ \\
\hline $0 / 1 V$ & 1,091 & OXYY & $1 / 14$ & $\circ \pi \Delta 9$ & $1 / 191$ & 0 Xty & $1, \pi \circ 4$ & $1 / 0$ & $\alpha_{r !}^{(r)}$ \\
\hline
\end{tabular}

\begin{tabular}{|c|c|c|c|c|c|c|c|c|c|}
\hline \multicolumn{4}{|c|}{$m=\Delta^{\prime} \circ$} & \multicolumn{4}{|c|}{$m=100$} & \multirow[b]{3}{*}{ مقدار واقعى } & \multirow[b]{3}{*}{ يارامتر } \\
\hline \multicolumn{2}{|c|}{ (ب) } & \multicolumn{2}{|c|}{ (الف) } & \multicolumn{2}{|c|}{ (ب) } & \multicolumn{2}{|c|}{ (الف) } & & \\
\hline $\mathrm{SE}$ & برآورد & $\mathrm{SE}$ & برآورد & $\mathrm{SE}$ & برآورد & $\mathrm{SE}$ & برآورد & & \\
\hline سזוס & $0, \gamma \circ 9$ & $0 / 1 r q$ & $0,19 V$ & $0 \times \wedge 9$ & optry & . & orVA & 01 & $\beta_{0}^{(1)}$ \\
\hline 0,049 & $1,0 V V$ & 0,091 & $1, \circ \wedge \Lambda$ & 0,194 & $|M| F$ & 0,190 & $0,9 V r$ & $1 / 0$ & $\beta_{1}^{(1)}$ \\
\hline 0,11 & 1,014 & $0,0 V T$ & $1,04 \lambda$ & $0, \gamma \wedge 9$ & WG4 & $0,14 \lambda$ & WTM & $1 / 0$ & $\lambda_{1}$ \\
\hline 0,099 & $1 / \%$ TA & $\circ \circ \Delta V$ & 1,049 & 0,401 & 0,914 & גונ & l,oVG & $1 / 0$ & $\sigma^{r}$ \\
\hline 0,0 D & ו ו & 0,041 & $-0 / 1 \wedge$ & $\circ, \wedge \vee$ & $-0, Y_{10}$ & 0,099 & $-0,1 r q$ & $-0 / 1$ & $\beta_{0}^{(r)}$ \\
\hline 0,019 & $1,0 V V$ & $\circ, 0 \Delta \Lambda$ & $1 / 019$ & 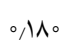 & $1 / 110$ & 0,119 & $1 / 0 \mathrm{H}$ & $1 / 0$ & $\beta_{1}^{(r)}$ \\
\hline 0,041 & -DIT & $\%$ o DT & $0,4 q 4$ & 0,11 & $\circ$ NIt & ONYY & سזو, & $\circ$ & $\lambda_{T}$ \\
\hline $0,0 \mathrm{rV}$ & rמקt & $\%$ YI & $\circ / D \circ Y$ & $\circ / \wedge \uparrow$ & $\circ / \Delta Y \Lambda$ & 0,049 & OPVG & $\circ$ & $\sigma_{*}^{r}$ \\
\hline $0, \pi 01$ & $1,0 V 0$ & $0 / 101$ & $1,0 \wedge 1$ & س & $1 / 104$ & OTYG & $y \circ \circ V$ & $1 /{ }^{\circ}$ & $\beta_{0}^{(r)}$ \\
\hline 0 olvt & 0994 & 0 & $1,04 \lambda$ & 0 TOS & 0,911 & $\circ, r^{\circ} V$ & 1,004 & $1 / 0$ & $\beta_{1}^{(r)}$ \\
\hline $0, \pi 01$ & $-0,109$ & $0 / 1 \mathrm{r}$ & $-0 / 10 \circ$ & oxtry & $-0,11$ & 0,190 & $-\circ \wedge \Delta \lambda$ & $-0 / 1$ & $\gamma_{0}^{(1)}$ \\
\hline 0,199 & $-r_{/} \circ \wedge \Delta$ & $0 \lambda 1 V$ & $-r / 0 \wedge 9$ & שצx & $-Y, K I Y$ & 0 TYY & $-1 / 9 k 4$ & $-r_{/}$ & $\gamma_{1}^{(1)}$ \\
\hline 0,044 & $1 / 014$ & $0,0 \wedge 1$ & 1,oDr & $\circ \lambda \Lambda$ & $M K A$ & $0 / \mathrm{Vr}$ & 1,099 & $1 / 0$ & $\gamma_{0}^{(r)}$ \\
\hline$\circ \% \mathrm{VD}$ & $\circ 911$ & 0,090 & $1 / 0 \Delta r$ & $0, \pi 19$ & 0,114 & $0 / 111$ &.$/ 119$ & $1 / 0$ & $\gamma_{1}^{(r)}$ \\
\hline 0,090 & .990 & 0,090 & 1,001 & $0 \lambda \vee \wedge$ & $1 / 401$ & $\circ, Y_{0} \circ$ & מוT & $1 / 0$ & $\alpha_{11}^{(r)}$ \\
\hline $0,0 Y 1$ & 0914 & 0,0 HY & 0,919 & $0 / 1 V$ &.$/ 190$ & $0,09 V$ & . 9人1 & $1 /{ }^{\circ}$ & $\alpha_{r !}^{(r)}$ \\
\hline
\end{tabular}

r r.r

در مدل (؟) متغير بيوسته از توزيع نرمال و متغير ترتيبى از توزيع جندجمالهاى آماسيده در صفر توليدمىشوند.

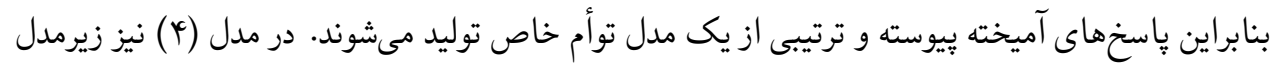




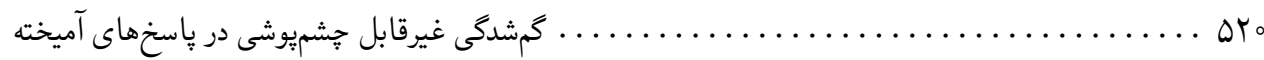

\begin{tabular}{|c|c|c|c|c|c|c|c|c|c|}
\hline \multicolumn{4}{|c|}{ كرار شبيهسازى } & \multicolumn{4}{|c|}{$m=\Delta^{\circ}$} & \multirow[b]{3}{*}{ مقدار واقعى } & \multirow[b]{3}{*}{ يارامتر } \\
\hline \multicolumn{2}{|c|}{ (ب) } & \multicolumn{2}{|c|}{ (الف) } & \multicolumn{2}{|c|}{ (ب) } & \multicolumn{2}{|c|}{ (الف) } & & \\
\hline $\mathrm{SE}$ & برآورد & $\mathrm{SE}$ & برآورد & SE & برآورد & $\mathrm{SE}$ & برآورد & & \\
\hline $0 \% V Y$ & oगा & $\circ / \wedge \Lambda$ & 0,111 & olve & $0, \pi \circ 1$ & 0,101 & 0 & 01 & $\beta_{0}^{(1)}$ \\
\hline $0 / 0 \mathrm{NT}$ & 0,994 & 0,090 & 0,949 & 0, & $0,9 \mathrm{VA}$ & 0,119 & 0,991 & $\circ$ & $\beta_{1}^{(1)}$ \\
\hline $0,0 \mathrm{VI}$ & 1,091 & 0,090 & 1/100 & $0 / 199$ & $\circ M 9$ & 0,144 & $0,94 \pi$ & $1 / 0$ & $\lambda_{1}$ \\
\hline 0,101 & $1,0 Y 1$ & $0,0 \mathrm{VI}$ & 1,0 TD & 0,101 & $0,94 Y$ & $0 / 109$ & $0,94 \pi$ & $1 /{ }^{\circ}$ & $\sigma^{r}$ \\
\hline 0,090 & $0 / 00$ & 0,101 & $0,14 \mathrm{Y}$ & $0, \pi \mid k$ & o & $0, \pi 10$ & rir & $0 / 1$ & $\beta_{0}^{(\digamma)}$ \\
\hline $0 / \wedge \mathrm{V}$ & ० 949 & 0,094 & 0,991 & $0, \pi 01$ & $1, \pi \%$ & 0 MrA & WV。 & $1 / 0$ & $\beta_{\uparrow}^{(\Gamma)}$ \\
\hline 0,109 & $-0,09 r$ & $0 / 109$ & $-0,090$ & $0, \pi \circ 0$ & $-0,1 \mu G$ & س & $-0, \pi 11$ & $-0 / 1$ & $\gamma_{0}^{(1)}$ \\
\hline 0,094 & $-r / 0 M \Lambda$ & 0,090 & $-r / 0 \Delta 9$ & ס & $-1, \lambda q 4$ & 0 Hr. & $-1 /, 1 M$ & $-r_{/ 0}$ & $\gamma_{1}^{(1)}$ \\
\hline $00 \wedge 0$ & $\circ 9 \circ \psi^{4}$ & 0,094 & oNva & $\circ \pi$ & 0,199 & $0, Y \circ T$ & $0,1 \Delta 9$ & $1 / 0$ & $\gamma_{0}^{(r)}$ \\
\hline 0,094 & $1,0 \wedge 0$ & $0 / \wedge \wedge$ & 1,091 & $0, \pi \mu 1$ & $|, \pi| \mid$ & $0, \pi r q$ & W & $1 / 0$ & $\gamma_{1}^{(r)}$ \\
\hline $0 / \Delta V$ & 1,001 & $0 / 190$ & 1,091 & $0, \pi 91$ & WTr & $0, \mu N V$ & $1, \pi 94$ & 1,0 & $\alpha_{1 !}^{(r)}$ \\
\hline $0,0 \Delta r$ & $1,01 \mathrm{~V}$ & 0,044 & 1,048 & $0, \lambda M$ & $0,94 Y$ & OKTG & ogGY & $1 / 0$ & $\alpha_{r 1}^{(r)}$ \\
\hline
\end{tabular}

\begin{tabular}{|c|c|c|c|c|c|c|c|c|c|}
\hline \multicolumn{4}{|c|}{$m=v \Delta$} & \multicolumn{4}{|c|}{$m=\Delta^{\circ}$} & \multirow[b]{3}{*}{ مقدار واقعى } & \multirow[b]{3}{*}{ بِارامتر } \\
\hline \multicolumn{2}{|c|}{ (ب) } & \multicolumn{2}{|c|}{ (الف) } & \multicolumn{2}{|c|}{ (ب) } & \multicolumn{2}{|c|}{ (الف) } & & \\
\hline $\mathrm{SE}$ & برآورد & SE & برآورد & SE & برآورد & SE & برآورد & & \\
\hline 0,091 & 0,119 & 0 गार & 0 orर & O MKT & $0, M V$ & $0, \pi 99$ & $0, \pi \vee \wedge$ & 01 & $\beta_{0}^{(1)}$ \\
\hline $0,0 \mathrm{Vr}$ & $\circ \Delta \Lambda_{0}$ & $0,0 \Delta r$ & $\circ / \Delta M \Lambda$ & $0 / \mathrm{VI}$ & 0,914 & $0, \pi Y \Lambda$ & $0,99 \mathrm{~V}$ & $\circ$ & $\beta_{1}^{(1)}$ \\
\hline $0,0 H Y$ & 1,010 & 0,0 & $1,0 \% \pi$ & odro & 1,019 & $0 / 0 \mathrm{~V}$ & $|M K|$ & $1 / 0$ & $\lambda_{1}$ \\
\hline 0,041 & $1,0 \%$ & $0 \% \mathrm{VI}$ & 1,0 Y० & $0 / 0 \wedge$ & $|/ T|$ & 0,109 & h\%。 & $1 /{ }^{\circ}$ & $\sigma^{r}$ \\
\hline $0, \lambda \mathrm{Vr}$ & 0,491 & $0, \psi_{0}$ & $0, T \wedge V$ & 0 H人s & $0,4 \circ 9$ & $0, \pi 94$ & 萑 & $0 / 1$ & $\beta_{0}^{(\Upsilon)}$ \\
\hline $0 \pi \mid r$ & $-0,999$ & $0, \pi \circ \Delta$ & $-0,919$ & $0, \pi \wedge 9$ & -0 NIV & OMKY & -oNrq & $-0 / \Delta$ & $\beta_{1}^{(r)}$ \\
\hline 0 olfo & 0,994 & 0,149 & $0,4 a Y$ & $0, \pi Y q$ & $\circ, N \circ r$ & $0, \pi 10$ & 0,994 & 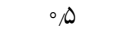 & $\lambda_{Y}$ \\
\hline 0,109 & 0,949 & 0 गार & $0,9 \wedge \mathrm{V}$ & $0, \pi \circ 9$ & $0, g \mu^{\prime}$ & 0,149 & $0, y \circ 9$ & $\circ$ & $\sigma_{*}^{\Upsilon}$ \\
\hline $0,09 V$ & $0,19 V$ & 0,109 & $0 / 1 v a$ & $0, \pi 91$ & $0, T Y Y$ & $0, \pi \wedge 9$ & $0, \pi 01$ & $0 / 1$ & $\beta_{0}^{(\Gamma)}$ \\
\hline 0 0 110 & $r / \lambda \circ 1$ & 0,099 & r,०94 & oNTS & T,KIF & $0 / 109$ & $r, \Gamma \circ 1$ & $r / 0$ & $\beta_{1}^{(\Gamma)}$ \\
\hline 0,090 & $-0,1 \mu$ & $0 / 0 \%$ & $-0,1 r q$ & TrT & $-0, \Gamma_{01}$ & TKM & $-0, \lambda \uparrow$ & $-0,1$ & $\gamma_{0}^{(1)}$ \\
\hline 0 . 110 & $-r / 0 V_{0}$ & $0,1 \circ \Lambda$ & $-r, 0 r q$ & 0,100 & $-r / T r q$ & سV & $-1, N \Delta T$ & $-r_{/ 0}$ & $\gamma_{1}^{(1)}$ \\
\hline $0,0 \mathrm{VI}$ & $1,0 \wedge 9$ & $0,0 \wedge r$ & $1,0 V r$ & -NQT & $1 / 1 \Lambda^{\circ}$ & $0 / 10 \Delta$ & $|/ 19|$ & $1 / 0$ & $\gamma_{0}^{(\Gamma)}$ \\
\hline 0,191 & $\circ 1199$ & $0, \pi \circ \Lambda$ & oNva & $0 \pi Y 1$ & $\circ, 9 \circ V$ & $0, \pi I 4$ & 0,190 & $1 / 0$ & $\gamma_{i}^{(r)}$ \\
\hline 0,190 & זrוגו & $0, \lambda M$ & WTS & $0, \pi \Delta \Delta$ & $|, \pi| \mid$ & $0, \pi 91$ & WrV & $1 / 0$ & $\alpha_{11}^{(r)}$ \\
\hline 0,090 & $\circ 9 V^{k}$ & $0,0 \wedge r$ & 0,994 & 0,100 & 0,199 & $0 / 1 Y$ & $\circ \wedge 90$ & $1 /{ }^{\circ}$ & $\alpha_{\Gamma 1}^{(r)}$ \\
\hline
\end{tabular}

مربوط به نسبت آميزنده پِاسخ ترتيبى فارغ از اثر تصادفى است و فرض مىشود

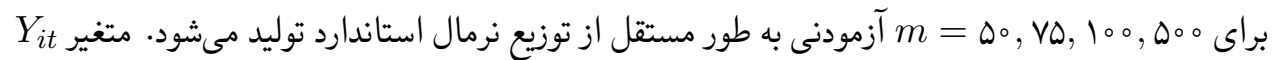


از توزيع نرمال با ميانكين

$$
\begin{aligned}
& \eta_{j, i t}=\frac{\exp \left(\theta_{j}-\left(\gamma_{\uparrow}^{(1)} X_{\backslash i t}+\lambda_{1} b_{i}^{(1)}\right)\right)}{1+\exp \left(\theta_{j}-\left(\gamma_{\uparrow}^{(1)} X_{1 i t}+\lambda_{1} b_{i}^{(1)}\right)\right)} j=\circ, 1 \\
& \pi_{i t}=\frac{\exp \left(\gamma_{0}^{(\Upsilon)}+\gamma_{1}^{(\Upsilon)} X_{1 i t}\right)}{1+\exp \left(\gamma_{0}^{(\Upsilon)}+\gamma_{1}^{(\Upsilon)} X_{\backslash i t}\right)}
\end{aligned}
$$

توليد مىشود. فرض مىشود امكان گمشدگى غيرتصادفى در ياسخ ترتيبى در زمان اول وجود دارد. احتمال

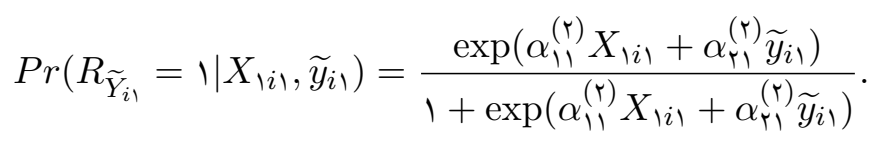

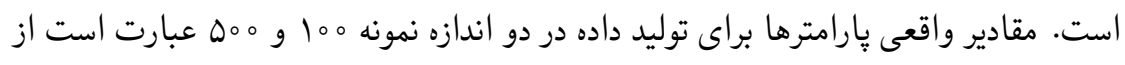
$\left(\beta_{0}, \beta_{1}, \theta_{1}, \gamma_{1}^{(1)}, \gamma_{0}^{(r)}, \gamma_{1}^{(r)}, \lambda_{1}, \sigma^{r}\right)=(0 / 1,1,0 / \Lambda, \circ / \Delta, 1,1,0, r \Delta, 1)$,

$$
\text { و در دو اندازه نمونه مه و VD عبارت است از }
$$

\begin{tabular}{|c|c|c|c|c|c|c|c|c|c|}
\hline \multicolumn{4}{|c|}{$m=\omega^{\circ \circ \circ}$} & \multicolumn{4}{|c|}{$m=100$} & \multirow[b]{3}{*}{ مقدار واقعى } & \multirow[b]{3}{*}{ يارامتر } \\
\hline \multicolumn{2}{|c|}{ (ب) } & \multicolumn{2}{|c|}{ (الف) } & \multicolumn{2}{|c|}{ (ب) } & \multicolumn{2}{|c|}{ (الف) } & & \\
\hline SE & برآورد & $\mathrm{SE}$ & برآورد & $\mathrm{SE}$ & برآورد & $\mathrm{SE}$ & برآورد & & \\
\hline $0 / 111$ & $0,11 \pi$ & 0,090 & $0 / 194$ & $0,49 \Lambda$ & $0, \pi 11$ & 0,400 & $0, \pi 40$ & 0,10 & $\beta$ 。 \\
\hline 0,090 & 0,914 & $0,0 V G$ & $\circ / \Delta \wedge G$ & o,TUT & $0, g r q$ & 0,419 & $0,9 \vee \wedge$ & 1,00 & $\beta_{1}$ \\
\hline $0 / 0 \mathrm{Vq}$ & OTKY & $0,0 \mathrm{VT}$ & ס ג & 0,119 & $0, \pi \mid 4$ & 0190 & ט D & $0, \pi \Delta$ & $\lambda_{1}$ \\
\hline$\circ,{ }^{\circ} \wedge \mathrm{V}$ & $1,0 \vee 9$ & $\circ / 0 \Delta \wedge$ & 1,oor & $0 / 119$ & 0941 & $0 / 1 V$ & 0,900 & $1 / 00$ & $\sigma^{r}$ \\
\hline 0,099 & $0 / 1 \circ 4$ & $0 / 1 V$ & .119 & $0 / 191$ & - ATA & OTYA & $0 / 110$ & $\circ \wedge \circ$ & $\theta_{1}$ \\
\hline $0 / 100$ & $\circ / 011$ & $\circ,{ }^{\circ} \wedge$ & -DTV & $0 / 199$ & OAYV & O KAS & $0,9 \wedge 1$ & $\circ \Delta \circ$ & \\
\hline $0 / 109$ & $\circ / V \Delta I$ & $\% / 0 \wedge 9$ & - $99 \wedge$ & & $\circ$ NIF & $\circ \mu$ & $\circ / \Delta \Delta r$ & 1,00 & \\
\hline 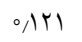 & \৯。 & $0 / 109$ & 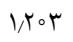 & $0, r 9 r$ & MAr & צTYK & $\not / \circ \Lambda$ & $1 / 00$ & $\gamma_{1}^{(1)}$ \\
\hline $0 / 0 / 191$ & $\circ / 0 \circ 9$ & $0, \pi I V$ & $0,49 V$ & 1 & $\circ \Delta \Delta \Lambda$ & $0 \times 4 V$ & $0,4 \wedge 1$ & $\circ \Delta \circ$ & $\alpha_{11}^{(r)}$ \\
\hline 0,119 & $0,19 V$ & oxmy & $0, \lambda V k$ & $0, \mu Y \Lambda$ & $\circ, \gamma \circ V$ & oxty & $0,5 \circ 9$ & $0 / 10$ & $\alpha_{r \mid}^{(r)}$ \\
\hline
\end{tabular}

$\left(\beta_{\circ}, \beta_{1}, \theta_{1}, \gamma_{1}^{(1)}, \gamma_{0}^{(r)}, \gamma_{1}^{(r)}, \lambda_{1}, \sigma^{r}\right)=(0 \lambda, \circ \Delta, \circ \wedge, \circ \Delta, 1,1, \circ, r \Delta, 1)$.

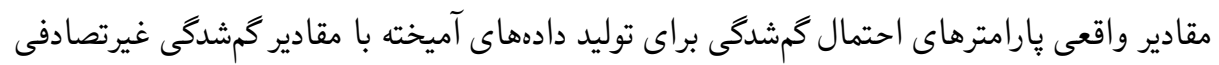

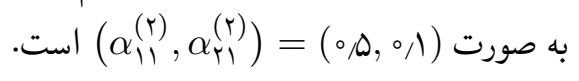


r

\begin{tabular}{|c|c|c|c|c|c|c|c|c|c|}
\hline \multicolumn{10}{|c|}{ جدول 9. نتايج شبيهسازى بخش Y.r با ه ه م ا تكرار شبيهسازى } \\
\hline \multicolumn{2}{|c|}{ 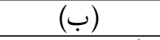 } & \multicolumn{2}{|c|}{ 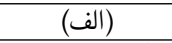 } & \multicolumn{2}{|c|}{ (ب) } & \multicolumn{2}{|c|}{ (الف) } & \multirow[b]{2}{*}{ مقدار واقعى } & \multirow[b]{2}{*}{ يارامتر } \\
\hline $\mathrm{SE}$ & برآورد & $\mathrm{SE}$ & برآورد & $\mathrm{SE}$ & برآورد & $\mathrm{SE}$ & برآورد & & \\
\hline $0, \pi 19$ & $0, \pi V G$ & $0, \pi 91$ & $0, \pi \Delta 9$ & $0, \pi \mathrm{Vq}$ & $0 \times 90$ & $0, \pi 9 \mathrm{~V}$ & $0, \pi M$ & $0 / 0$ & $\beta$ 。 \\
\hline $0,0 V_{0}$ & $0,9 \circ 4$ & $0,0 \mathrm{VG}$ & 0,090 & $0, \pi \times 9$ & $0, g 41$ & $0, \pi T_{0}$ & 0,990 & $\circ / \Delta \circ$ & $\beta_{1}$ \\
\hline 0,111 & $0, \pi \Delta V$ & $0 / 119$ & ג & $\circ, \pi \circ \Delta$ & O TAV & $0 / K 4$ & OMTY & $0, T \Delta$ & $\lambda_{1}$ \\
\hline $0,1 K 1$ & - ATS & $0, N Y$ & 1,099 & $0,1 \mathrm{~V}$ & $1, r \circ 4$ & $0 / V_{0}$ & $1, \lambda \psi_{0}$ & 1,00 & $\sigma^{r}$ \\
\hline ס & OAYT & 0,100 & - 944 & س & $0 \wedge 99$ & $0, r_{0} 1$ & $0,9 \circ \gamma$ & $0 / \wedge 0$ & $\theta_{1}$ \\
\hline $0 / 0 \wedge 9$ & $\circ \Delta \wedge Y$ & $0,0 \wedge 1$ & $\circ \Delta \wedge q$ & $0, \mu K V$ & $0,91 Y$ & $0, \pi T \Delta$ & 0,991 & $\% / 0$ & $\gamma_{1}^{(1)}$ \\
\hline 0,490 & $0 \Delta Y 4$ & 0 TAY & $\circ, 90 \mathrm{r}$ & 0,11 & 0,910 & $0, \mu r$ & $0,9 \circ 4$ & 1,00 & $\gamma_{0}^{(r)}$ \\
\hline$\circ, \mu \circ \Delta$ & $1, T \circ Y$ & ס & $1, \pi \circ 9$ & $0, \pi T 1$ & $1, \pi \wedge 。$ & $0, r v 4$ & $1,1,9$ & 1,00 & $\gamma_{1}^{(r)}$ \\
\hline 0,499 & $\circ \Delta T_{\circ}^{\circ}$ & $\circ \pi \circ 9$ & r & $0,4 \circ 9$ & 0,949 & $0, \mu \circ r^{2}$ & ו וצו & $\circ / \Delta \circ$ & $\alpha_{\lambda !}^{(r)}$ \\
\hline 0 - & $0, \pi 10$ & $0, \pi \wedge 9$ & $0, \pi 10$ & $0, \pi v \pi$ & $0, \pi$ & $0, \mu \Lambda$ & $0, \pi 91$ & $0 / 10$ & $\alpha_{r !}^{(r)}$ \\
\hline
\end{tabular}

به منظور برازش مدلهاى مورد بررسى به نمونهاى توليد شده، برآوردهاى بيشينه درستنمايى يارامترهاى

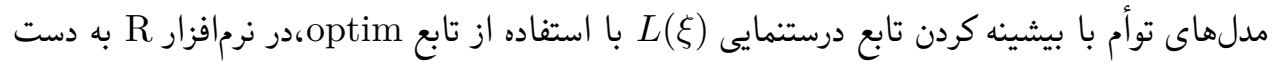

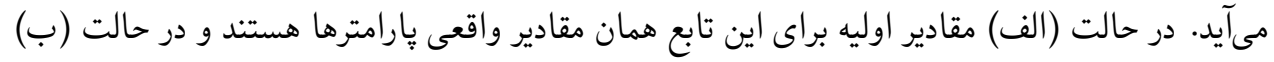
مقادير اوليه مقدارهاى تصادفى هستند. به عبارتى در حالت (ب)، در هر تكرار شبيهسازى با توليد اعداد

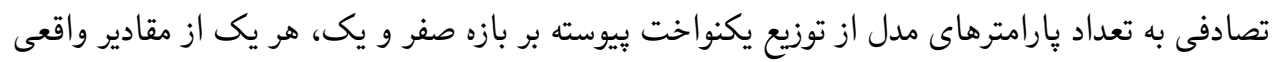

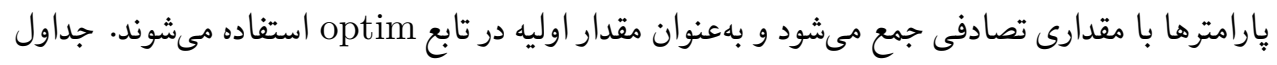

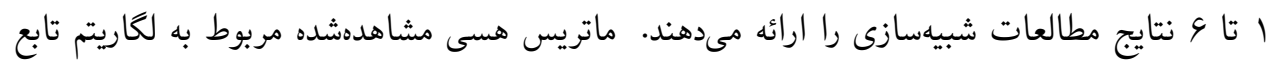

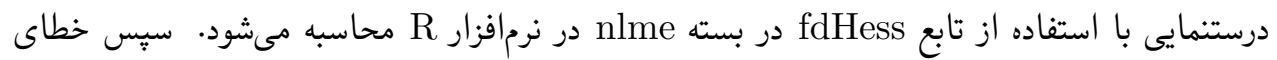

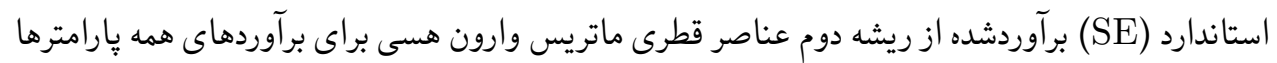

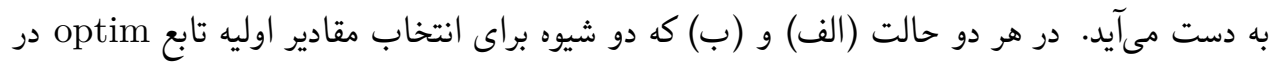

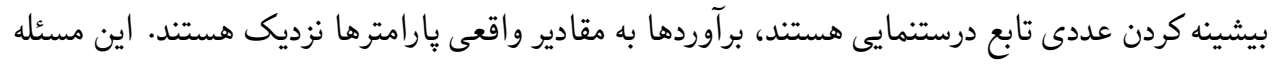

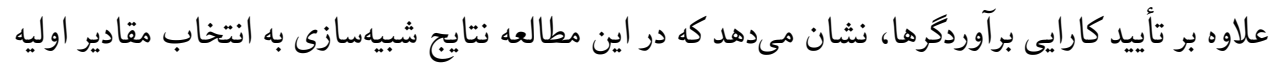

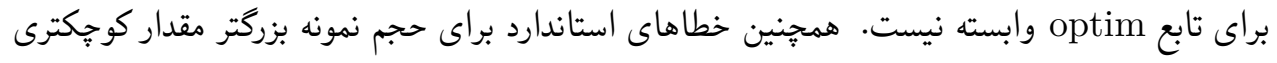

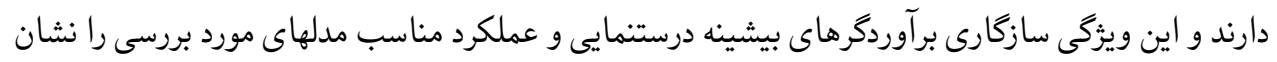


در اين بخش دو مجموعه داده واقعى تغييرات زندگى آمريكايىها ACL و آزمون موفقيت فردى بيبادى

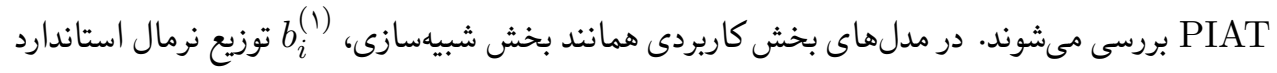
دارد. براى هر مدل معيار BIC و AIC

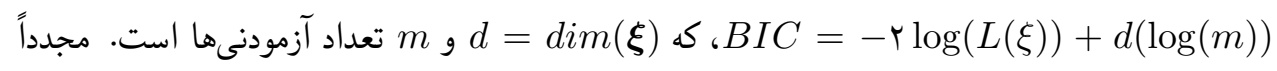

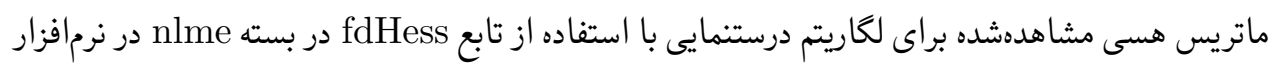

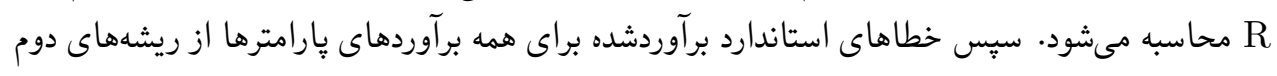
عناصر قطرى ماتريس وارون هسى محاسبه مىشوند. سئد

\section{ACL د Pادهoاى F.I}

سلسله مطالعه تغييرات زندكى آمريكايىها (ACL) يك مطالعه طولى ملى طولانىمدت است كه روى

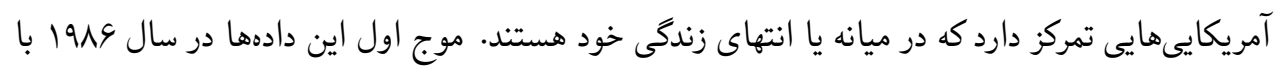

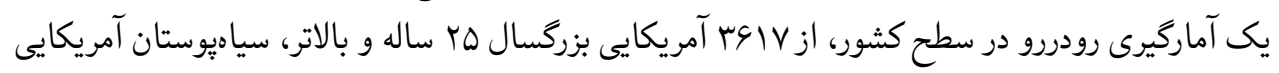

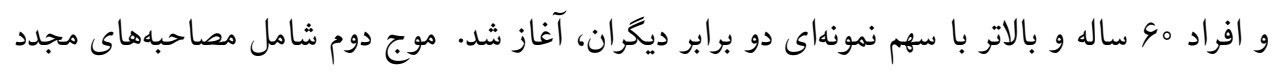

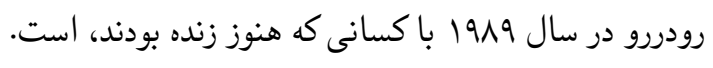

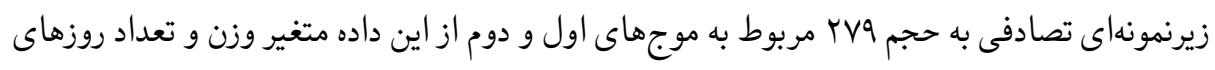

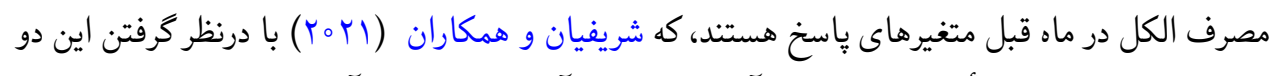

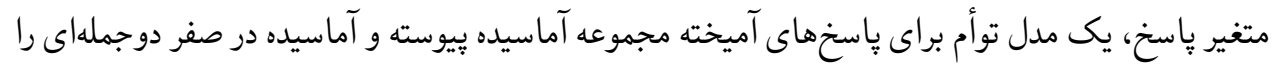

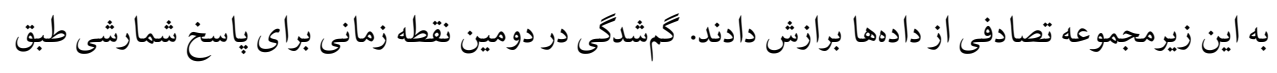

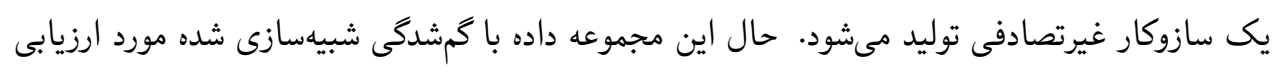

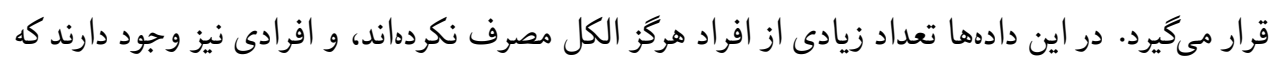

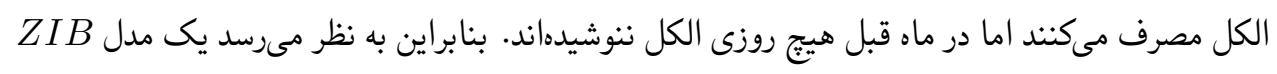

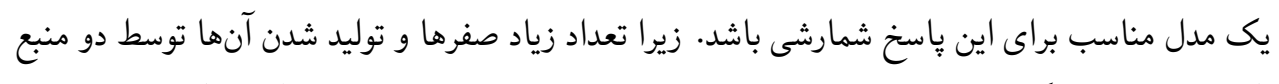

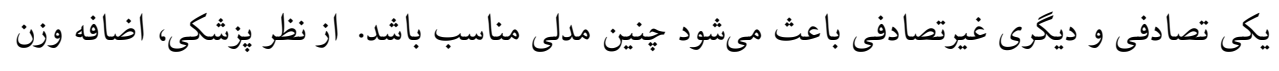

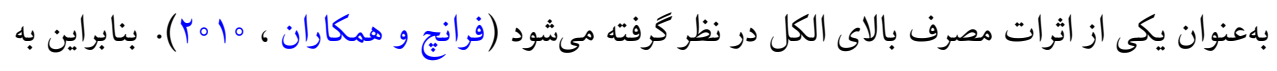

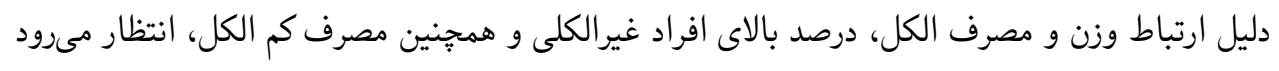




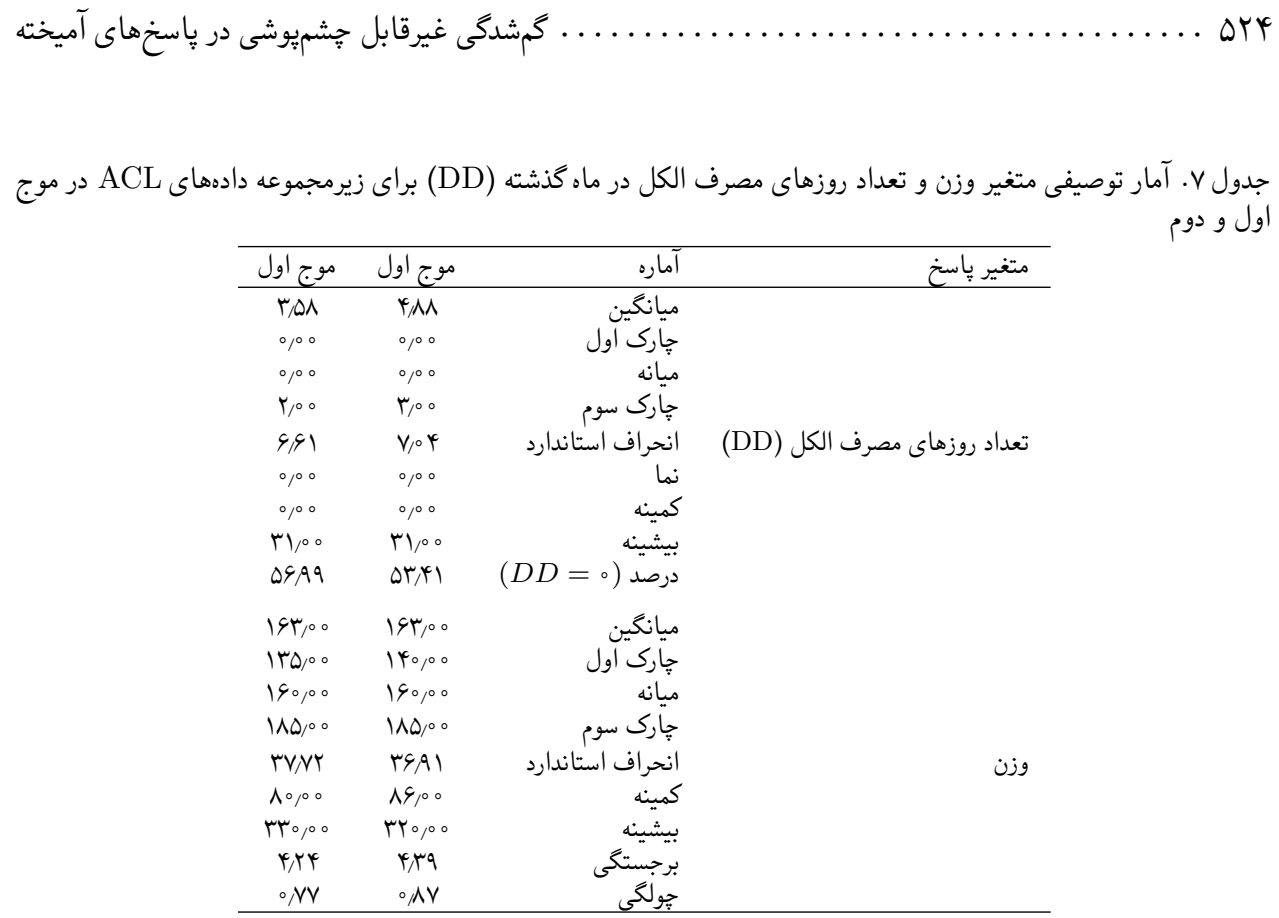

كه آماسيدكى در يك محدوده خاص از متغير وزن رخ دهد. ويثگىهاى توصيفى اين دو ياسخ در جدول V ارائه شده است.

با انجام يك آزمون نايارامترى كولموكروف-اسميرنوف و يك آزمون نسبت درستنمايى بوتاستريى

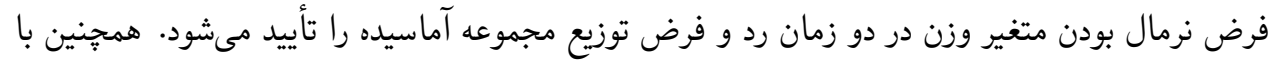

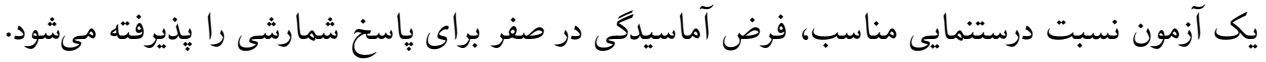

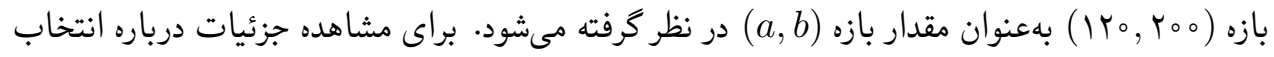

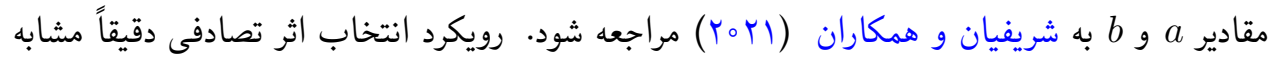

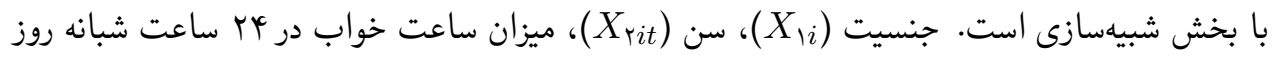

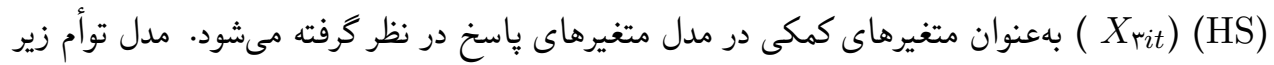


براى دو پاسخ آميخته طولى به همراه سازوكار گمدگى غيرقابل خشميوشى در نظر گرفته مىشود:

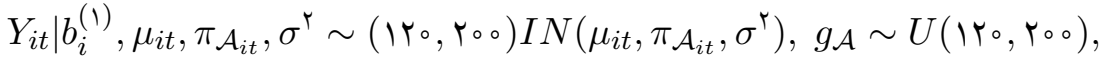

$$
\begin{aligned}
& \mu_{i t}=\beta_{\circ}^{(1)}+\beta_{1}^{(1)} X_{1 i}+\beta_{r}^{(1)} X_{r i t}+\beta_{r}^{(1)} X_{r i t}+\lambda_{1} b_{i}^{(1)} \text {, }
\end{aligned}
$$

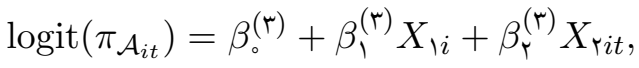

$$
\begin{aligned}
& \left.N_{i t} \mid b_{i}^{(।)}, \nu_{i t}, \pi_{\circ i t} \sim Z I B(\mu), \frac{\nu_{i t}}{1+\nu_{i t}}, \pi_{\circ i t}\right), \\
& \log \left(\nu_{i t}\right)=\gamma_{0}^{(1)}+\gamma_{\uparrow}^{(1)} X_{l i}+\gamma_{\Gamma}^{(1)} X_{\curlyvee i t}+\gamma_{\Gamma}^{(1)} X_{r i t}+\lambda_{1} b_{i}^{(1)} \text {, } \\
& \operatorname{logit}\left(\pi_{\circ i t}\right)=\gamma_{\circ}^{(r)}+\gamma_{i}^{(r)} X_{i i}+\gamma_{r}^{(r)} X_{r i t} \text {, }
\end{aligned}
$$

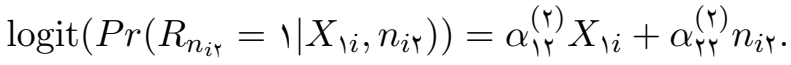

اكر در مدل (10) ضريب (Y)

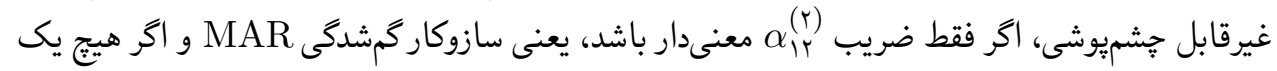

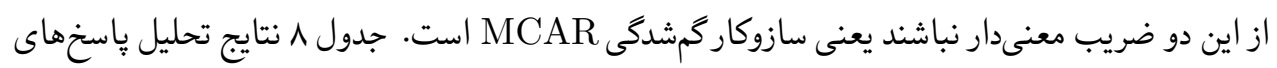

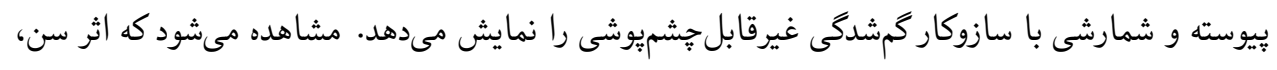

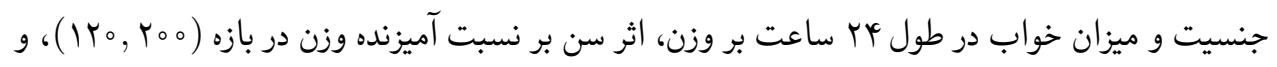

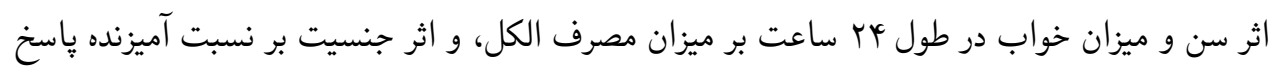

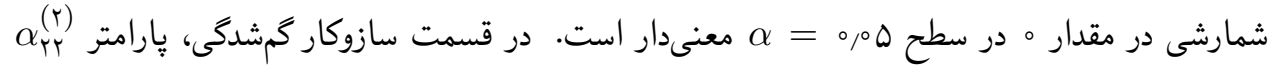

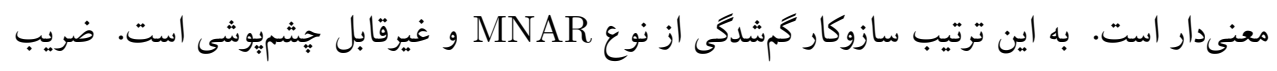

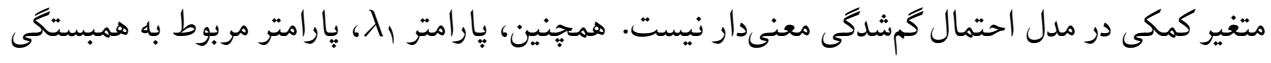

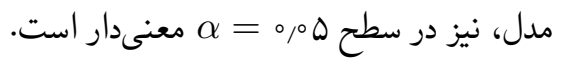

\section{PIAT دادهoاى P.Y}

دادههاى PIAT كه قبلاً توسط شريفيان و همكاران (Y.Y0) مورد تحليل قرار گرفته است، در بين سالهاى 1919 و 1999 جمع آورى شده و شامل مصاحبههاى دو سال يك بار از مادرها و فرزندهايشان

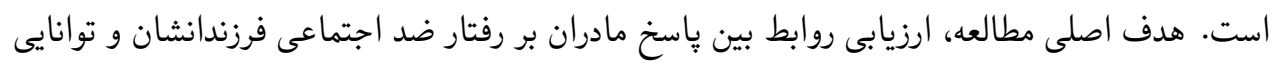

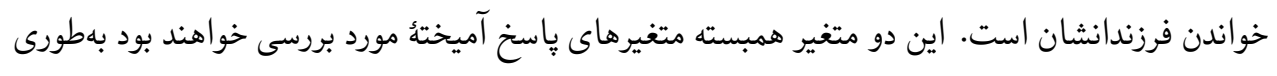


\&

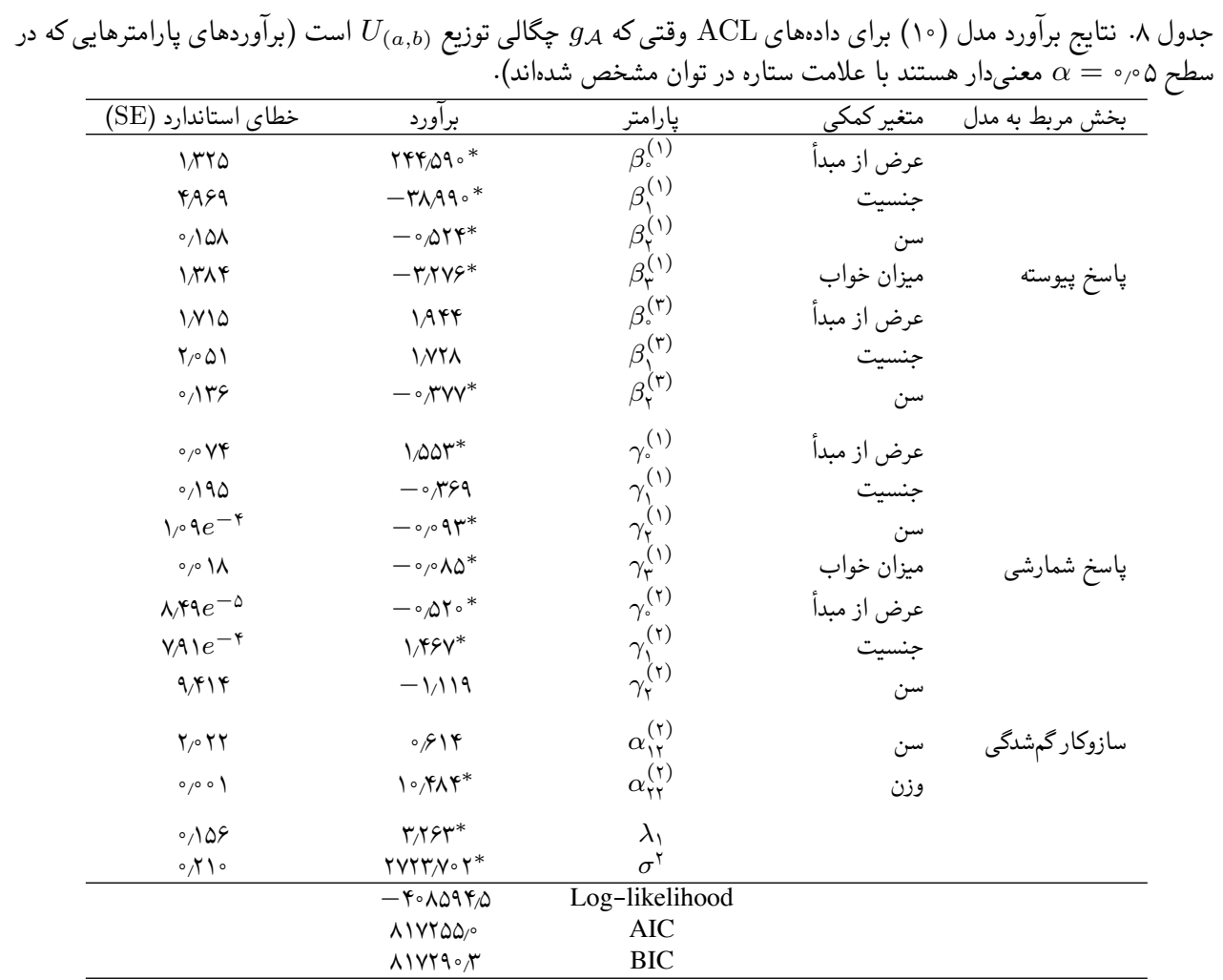

كه بِاسخ ترتيبى رفتار ضد اجتماعى فرزند (anti) و وِاسخ بيوسته توانايى خواندن فرزند (read) هستند.

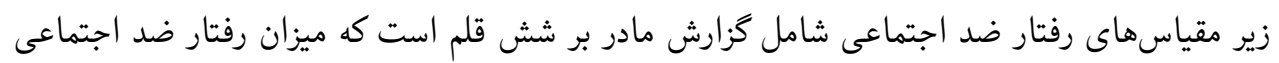

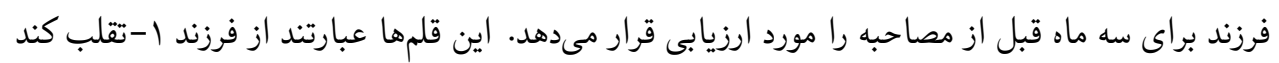

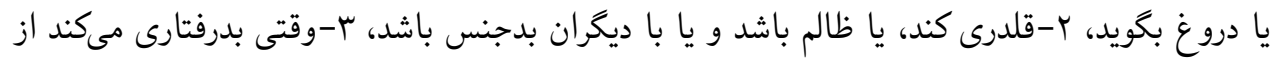

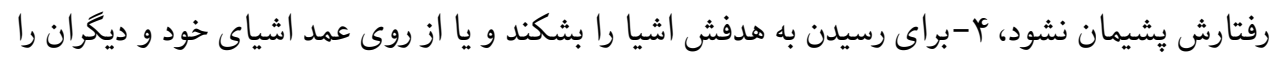

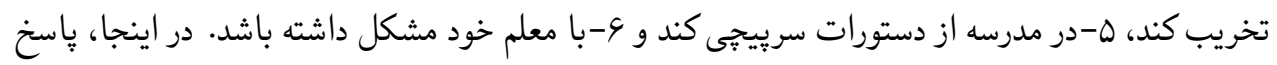

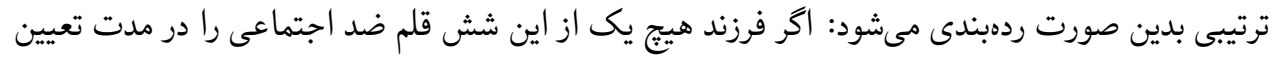

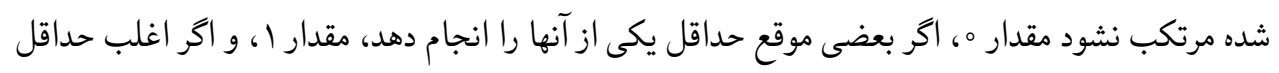

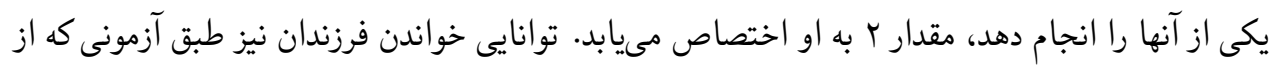

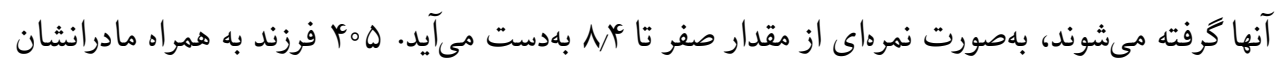




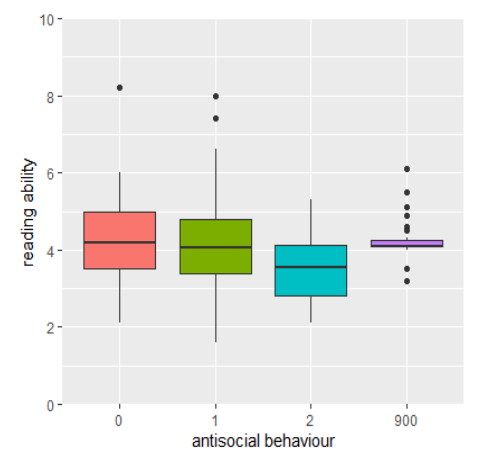

(ب)

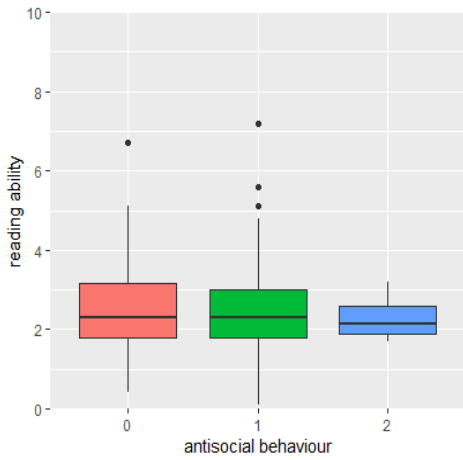

(الف)

شكل ا. نمودار جعبهاى توانايى خواندن براى سطوح مختلف رفتار ضد اجتماعى كودكان در الف- اولين دوره زمانى، ب- دومين دوره زمانى ندك

در اين مطالعه شركت كردهاند. در دوره اول مطالعه هيج يك از متغيرهاى ياسخ داراى گمشدىى نيستند ولى در دوره دوم مطالعه شدت ناهنجارى داراى گمشدىى است (1 درصد داده گمشده براى شدت ناهنجارى در دوره دوم). متغيرهاى ييشگو شامل جنسيت فرزندان (X)، دختر=ه و يسر=I )، سن فرزندان در زمان

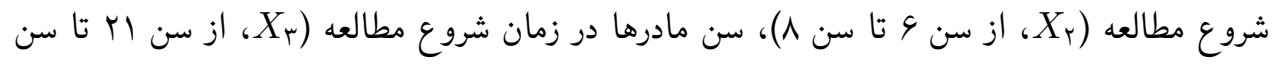
9ץ)، و زمان (r) مىشوند. شكل ا نمودار جعبهاى ياسخهاى توانايى خواندن براى مقادير مختلف از سطوح رفتارهاى ضد اجتماعى را نشان مىدهد. بر اساس اين شكل، فرزندان با رفتارهاى ضد اجتماعى كمتر داراى توانايى خواندن بيشترى نسبت به فرزندانى با رفتارهاى ضد اجتماعى بيشتر در تمام دورههاى زمانى تعيين شده

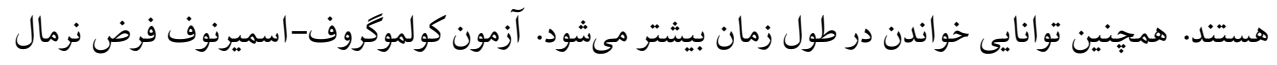
بودن متغير توانايى خواندن را تأييد مىكند. همجنين فرض آماسيدكى ياسخ ترتيبى، رفتار ضد اجتماعى، در سطح دوم (مقدار () در دو زمان با استفاده از يك آزمون نسبت درستنمايى مناسب، تأييد مىشود

$$
\cdot(P-\text { value }<\text { ०,००० })
$$

مطلوب است كه همزمان با بررسى تأثير جنسيت كودك، سن كودى و سن مادر كودى، نوع گمشدگى در زمان دوم براى ياسخ anti نيز مشخص شود. بنابراين، مدل توأم زير براى دو ياسخ آميخته طولى به 
A

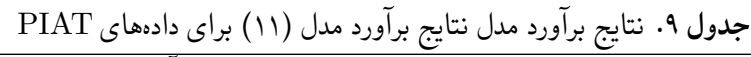

\begin{tabular}{|c|c|c|c|c|}
\hline خطاى استاندارد (SE) & برآورد & بِارامتر & متغير كمكى & بخش مربط به مدل \\
\hline $0, \wedge \wedge$ & o. akr* & $\beta_{\text {。 }}$ & عرض از مبدأ & \\
\hline $0 \% 1 Y$ & $-0,0 Y 1$ & $\beta_{1}$ & جنسيت & \\
\hline $0 \% V$ & 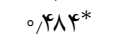 & $\beta_{r}$ & سن كُودى & بِاسخ يبوسته \\
\hline$\% \%$ & $0,1 \circ \gamma^{*}$ & $\beta_{r}$ & سن مادر كودى & \\
\hline$\% \Delta A$ & 1,099 & $\beta_{\psi}$ & زمان & \\
\hline 99 & $T y x_{0} \circ *$ & $\theta_{1}$ & عرض از مبدأ & \\
\hline $0, \pi 49$ & $-0, \wedge r 4^{*}$ & $\gamma_{1}^{(1)}$ & جنسيت & \\
\hline 0,049 & $0, \mu r v^{*}$ & $\gamma_{r}^{(1)}$ & سن كودى & \\
\hline 0019 & $-0,0 \wedge r$ & $\gamma_{r}^{(1)}$ & سن مادر كورى & ي إسخ ترتيبى \\
\hline$\% Y_{0}$ & $0,194 *$ & $\gamma_{*}^{(1)}$ & زمان & \\
\hline 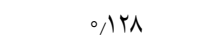 & $0, \mu 4 y^{*}$ & $\gamma_{o}^{(\uparrow)}$ & عرض از مبدأ & \\
\hline $0 \% Y$ & $-0,0 \Lambda^{*}$ & $\gamma_{1}^{(r)}$ & جنسيت & \\
\hline o & $-0, \pi T \Delta$ & $\gamma_{r}^{(r)}$ & سن كودى & \\
\hline 0 0 TII & $\circ \wedge \Delta \wedge *$ & $\alpha_{1 \uparrow}^{(\uparrow)}$ & جنسيت & \\
\hline $0 \% T Y$ & orkKF* & $\alpha_{r r}^{(r)}$ & سن كودى & سازوكار گمشدىى \\
\hline $0,1 \mathrm{Vq}$ & $-0,1 \Lambda_{0}$ & $\alpha_{r r}^{(r)}$ & سن مادر كورى & \\
\hline 0,149 & orva* & $\alpha_{\mu \varphi}^{(r)}$ & رفتار ضد اجتماعى & \\
\hline \multirow{5}{*}{0,091} & $0,499 *$ & $\lambda_{1}$ & & \\
\hline & $0 \Delta \varphi^{*}$ & $\sigma^{r}$ & & \\
\hline & $-r \wedge r q 4 \lambda, 4$ & Log-likelihood & & \\
\hline & agVary A & $\begin{array}{l}\text { AIC } \\
\text { BIC }\end{array}$ & & \\
\hline & 9910109 & & & \\
\hline
\end{tabular}

همراه سازوكار گمشدگى غيرقابل جشميوشى در نظر گرفته مىشود:

$$
\begin{aligned}
& \operatorname{read}_{i t}=\beta_{\circ}+\beta_{\uparrow} X_{\backslash i}+\beta_{\Upsilon} X_{\Upsilon i}+\beta_{\Upsilon} X_{r i}+\beta_{\uparrow} X_{i t}+\lambda_{\uparrow} b_{i}^{(1)}+\epsilon_{i t} \\
& \operatorname{logit}\left(\eta_{\circ}, i t\right)=\theta_{\circ}-\left(\gamma_{1}^{(1)} X_{1 i}+\gamma_{\Gamma}^{(1)} X_{r i}+\gamma_{\Gamma}^{(1)} X_{r_{i}}+\gamma_{\varphi}^{(1)} X_{i t}+\lambda_{1} b_{i}^{(1)}\right) \text {, } \\
& \operatorname{logit}\left(\eta_{\backslash, i t}\right) \theta_{1}-\left(\gamma_{1}^{(1)} X_{1 i}+\gamma_{r}^{(1)} X_{r i}+\gamma_{r}^{(1)} X_{\Gamma_{i}}+\gamma_{\varphi}^{(1)} X_{i t}+\lambda_{1} b_{i}^{(1)}\right) \text {, } \\
& \operatorname{logit}\left(\pi_{i}\right)=\gamma_{0}^{(\Upsilon)}+\gamma_{1}^{(\digamma)} X_{l i}+\gamma_{\Gamma}^{(\digamma)} X_{\Upsilon i} \text {, }
\end{aligned}
$$

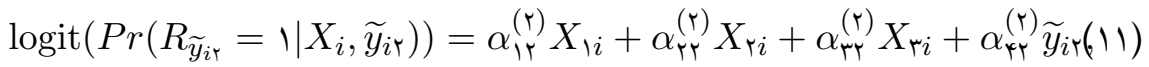

كه

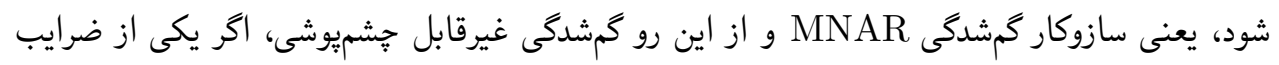

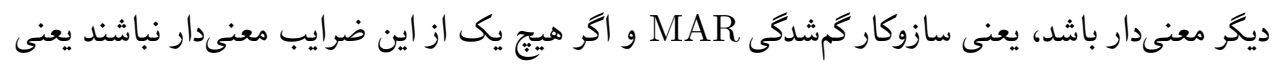


جدول ه نتايج تحليل باسخهاى بيوسته و ترتيبى با سازوكار گمشدى غيرقابلجشميوشى را نمايش

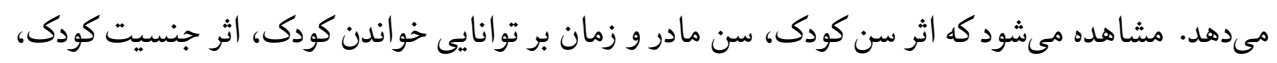

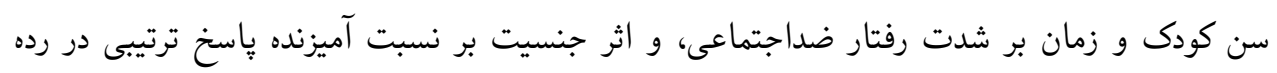

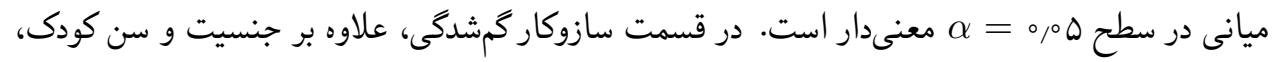

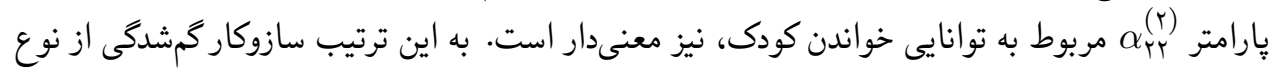

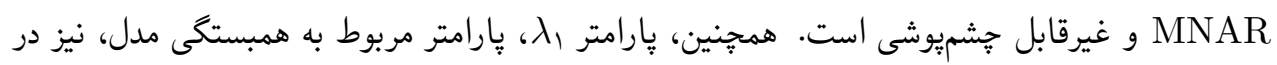
سطح ه ه $\alpha=0 / 1$ معنىدار است.

\section{بحث و نتيجهكيرى}

در اين مقاله سازوكار گمشدى غيرقابل جشميوشى در دو مدل توأم براى پاسخهاى آميخته مجموعه آماسيده

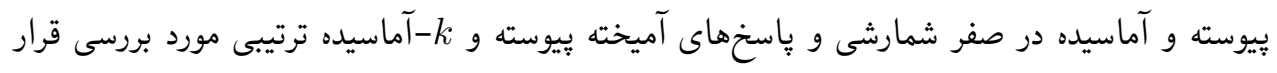

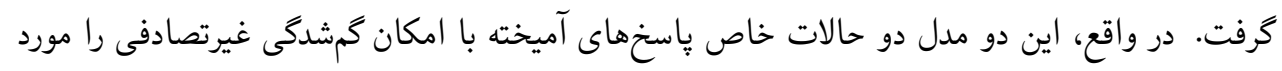

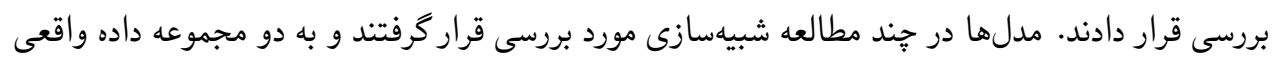

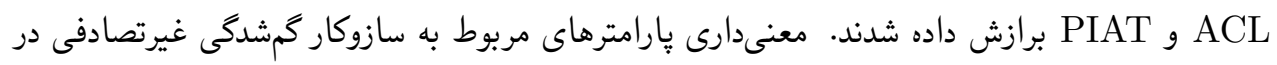

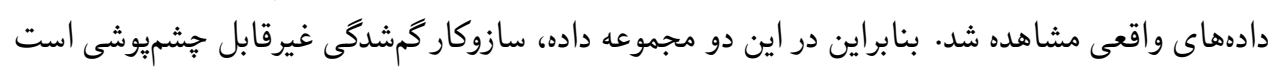

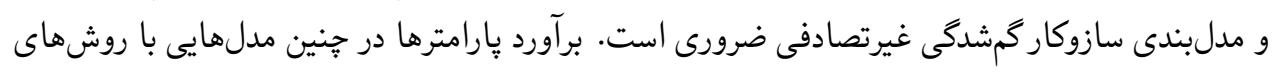

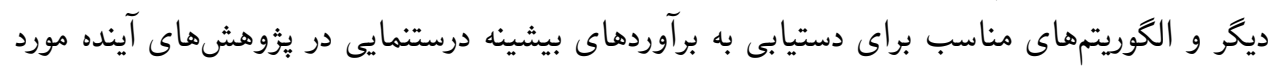
بررسى قرار مى ميرد.

\section{تقدير و تشكر}

از داوران و ويراستار محترم مجله كه با توصيههاى ارزشمند خود سبب ارتقاى اين مقاله و ارائه بهتر آن شدهاند كمال تشكر را داريم. 


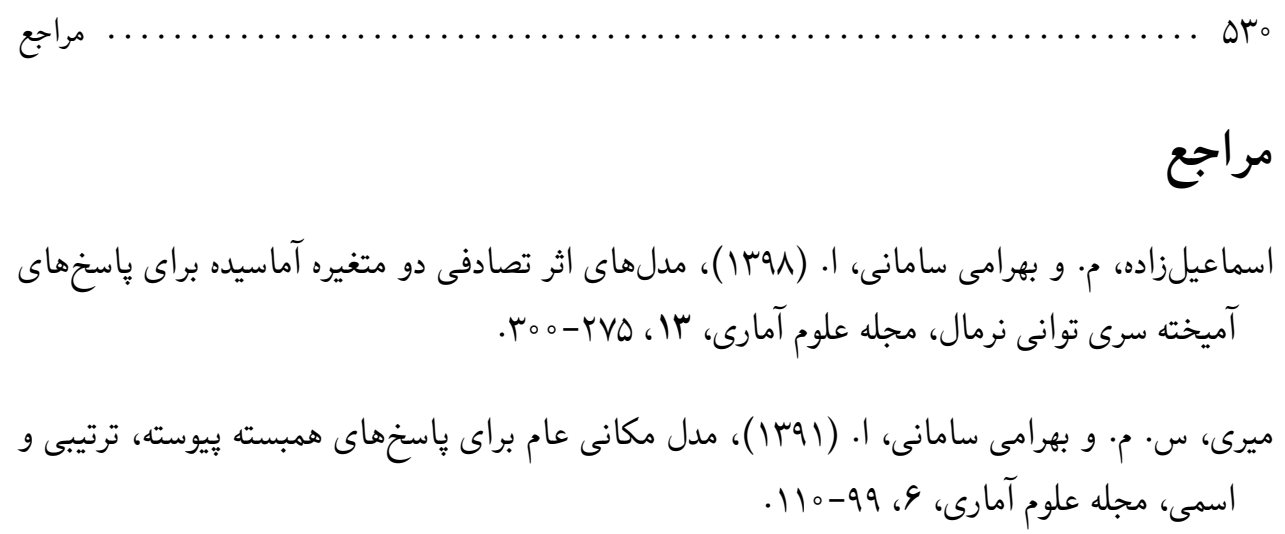

Bahrami Samani, E., Ganjali, M. and Khodadadi, A. (2008), A Latent Variable Model for Mixed Continuous and Ordinal Responses, Journal of Statistical Theory and Applications, 7, 337-349.

Bahrami Samani, E., and Ganjali, M. (2010), A Multivariate Latent Variable Mixed-Data from Continuous and Ordinal Responses with Possibility of Missing Responses, Application and Applied Mathematics: An international Journal (AAM), 5 (10), 1364-1584.

Bahrami Samani, E., Ganjali, M. and Eftekhari, S. (2010), A Latent Variable Model for Mixed Continuous and Ordinal Responses with Nonignorable Missing Responses: Assessing the Local Influence via Covariance Structure, Sankhya: The Indian Journal of Statistics, 27 (B), 38-57.

Bahrami Samani, E., and Tahmasebinejad, Zh. (2011), Joint Modeling of Mixed Correlated Nominal, Ordinal and Continuous Responses. Journal of Statistical Research, 45, 37-47.

Bahrami Samani, E., Ganjali, M., and Amirian, Y. (2012), Likelihood Estimation for Longitudinal Zero-Inflated Power Series Regression Models, Journal of Applied Statistic, 39, 1965-1974.

Bahrami Samani, E. (2013), Local Influence in Bayesian Elliptically 
Contoured-Ordinal Model for Mixed Data, Application and Applied Mathematics: An international Journal (AAM), 8, 391-403.

Bahrami Samani, E., and Ganjali, M. (2014), Mixed Correlated Bivariate Ordinal and Negative Binomial Longitudinal Responses with Nonignorable Missing Values, Comunications in Statistics-Theory and Methods, 43, 2059-2673.

De Leon, A. R., and Chough, K. C. (2013), Analysis of Mixed Data, Methods E) Applications, Taylor \& Francis.

French, M. T., Notron, E. C., and Fang, H. (2010), Alcohol Consumption and Body Weight, Health Economics, 19, 814-832.

Kelley, M. E., and Anderson, S. J. (2008), Zero Inflation in Ordinal Data: Incorporating Susceptibility to Response Through the Use of a Mixture Model, Statistics in Medicine, 27, 3674-88.

Lambert, D. (1992), Zero-Inflated Poisson Regression, with an Application to Defects in Manufacturing, Technometrics, 34, 1-14.

Little, R. J. and Rubin, D. (2002), Statistical Analysis with Missing Data. Second Edition, New York, Wiley.

Little, R. J., and Schluchter M. (1985), Maximum Likelihood Estimation for Mixed Continuous and Categorical Data with Missing Values, Biometrika, 72, 497-512.

Mc Culloch, C. (2007), Joint Modeling of Mixed Outcome Type Using Latent Variables, Statistical Methods in Medical Research, 17, 53-73.

Razie, F., Bahrami Samani, E., and Ganjali, M. (2017), Latent Variable Model for Mixed Correlated Power Series and Ordinal Longitudinal Re- 
sponses with Nonignorable Missing Values, Journal Communication in Statistics-Theory and Methods, 46, 5738-5753.

Razie, F., Bahrami Samani, E., and Ganjali, M. (2019), Analysis of Mixed Longitudinal (k,l)-Inflated Power Series, Ordinal and Continuous Responses with Sensitivity Analysis to Non-Ignorable Missing Mechanism, Journal Communication in Statistics-Simulation and Computation, 1-27. doi: 10.1080/03610918.2019.1601215

Sharifian, N., Bahrami Samani, E., and Ganjali, M. (2021), Joint Modeling for Longitudinal Set-Inflated Continuous and Count Responses, Communications in Statistics-Theory and Methods, 50, 1134-1160.

Sharifian, N., Bahrami Samani, E., and Ganjali, M. (2020), Joint Model for Longitudinal Mixture of Normal and Zero-Inflated Power Series Correlated Responses, Journal of Biopharmaceutical Statistics, 1-24. doi:10.1080/10543406.2020.1814798

Teixeira-Pinto, A., and Normand, S. L. T. (2009), Correlate Continuous and Binary Outcomes: Issues and Applications, Statistics in Medicine, 28, 1753-1773.

Wang, W. (2013), Identifiability of Linear Mixed Effects Models, Electronic Journal of Statistics, 7, 244-263.

Yang, Y. and Kang, J.(2010), Joint Analysis of Mixed Poisson and Continuous Longitudinal Data with Nonignorable Missing Values, Computational Statistics and Data Analysis, 54, 193-207. 
Journal of Statistical Sciences, Autumn and Winter, 2021

Vol. 15, No. 2, pp 505-531

DOI: $10.29252 /$ jss.15.2.505

\title{
Modeling of Continuous-Count and Continuous-Ordinal Mixed Longitudinal Bivariate Inflated Responses with Pos- sibility of Non Random Missingness
}

Sharifian, N. and Bahrami Samani, E.

Department of Statistics, Shahid Beheshti University, Tehran, Iran.

\begin{abstract}
One of the most frequently encountered longitudinal studies issues is data with losing the appointments or getting censoring. In such cases, all of the subjects do not have the same set of observation times. The missingness in the analysis of longitudinal discrete and continuous mixed data is also common, and missing may occur in one or both responses. Failure to pay attention to the cause of the missing (the mechanism of the missingness) leads to unbiased estimates and inferences. Therefore, in this paper, we investigate the mechanism of nonignorable missing in set-inflated continuous and zero-inflation power series, as well as the continuous and $k$-inflated ordinal mixed responses. A full likelihood-based approach is used to obtain the maximum likelihood estimates of the parameters of the models. In order to assess the performance of the models, some simulation studies are performed. Two applications of our models are illustrated for the American's Changing Lives (ACL) survey, and the Peabody Individual Achievement Test (PIAT) data set.
\end{abstract}

Keywords: Longitudinal Mixed data, Inflation, Random effects, Missing values.

Mathematics Subject Classification (2010): 62J12. 